\section{Federation University ResearchOnline}

\section{https://researchonline.federation.edu.au}

Copyright Notice

(C) 2019. This manuscript version is made available under the CC-BY-NC-ND 4.0 license http://creativecommons.org/licenses/by-nc-nd/4.0/

Guo, H, Ooi, E.T, Saputra, A.A, Yang, Z, Natarajan, S, Ooi, E.H, \& Song, C. (2019). A quadtree-polygon-based scaled boundary finite element method for image-based mesoscale fracture modelling in concrete. Engineering Fracture Mechanics, 211, 420441.

Which has been published in final form at:

https://doi.org/10.1016/j.engfracmech.2019.02.021 


\title{
A Quadtree-Polygon-Based Scaled Boundary Finite Element Method for Image-Based Mesoscale Fracture Modelling in Concrete
}

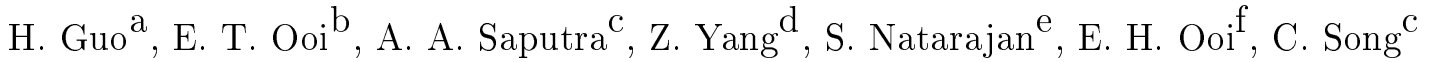 \\ ${ }^{a}$ School of Mining Engineering, Central South University, 410083, Hunan, China \\ ${ }^{\mathrm{b}}$ School of Science, Engineering 6 Information Technology, Federation University, Ballarat \\ VIC3350, Australia \\ ${ }^{\mathrm{c}}$ School of Civil \& Environmental Engineering, The University of New South Wales, Sydney NSW \\ 2032, Australia

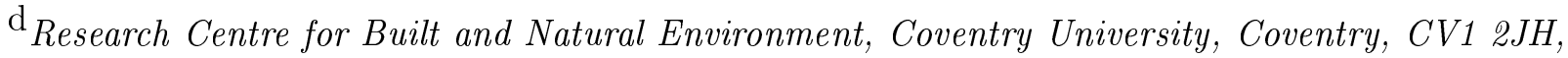 \\ United Kingdom \\ ${ }^{\mathrm{e}}$ Integrated Modelling and Simulation Lab, Department of Mechanical Engineering, Indian \\ Institute of Technology, Madras, Chennai, 600036, India

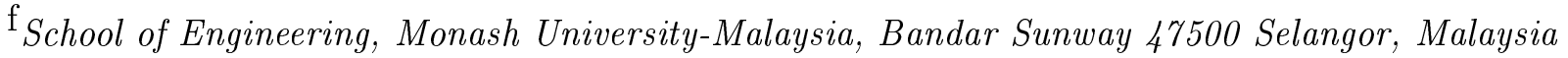

\begin{abstract}
A quadtree-polygon scaled boundary finite element-based approach for image-based modelling of concrete fracture at the mesoscale is developed. Digital images representing the two-phase mesostructure of concrete, which comprises of coarse aggregates and mortar are either generated using a take-and-place algorithm with a user-defined aggregate volume ratio or obtained from X-ray computed tomography as an input. The digital images are automatically discretised for analysis by applying a balanced quadtree decomposition in combination with a smoothing operation. The scaled boundary finite element method is applied to model the constituents in the concrete mesostructure. A quadtree formulation within the framework of the scaled boundary finite element method is advantageous in that the displacement compatibility between the cells are automatically preserved even in the presence of hanging nodes. Moreover, the geometric
\end{abstract}


flexibility of the scaled boundary finite element method facilitates the use of arbitrary sided polygons, allowing better representation of the aggregate boundaries. The computational burden is significantly reduced as there are only finite number of cell types in a balanced quadtree mesh. The cells in the mesh are connected to each other using cohesive interface elements with appropriate softening laws to model the fracture of the mesostructure. Parametric studies are carried out on concrete specimens subjected to uniaxial tension to investigate the effects of various parameters e.g. aggregate size distribution, porosity and aggregate volume ratio on the fracture of concrete at the meso-scale. Mesoscale fracture of concrete specimens obtained from X-ray computed tomography scans are carried out to demonstrate its feasibility.

Keywords: scaled boundary finite element method; meso-scale; fracture; quadtree; polygon; image-based analysis; concrete

\section{Introduction}

When concrete is observed at shorter length scales, it can be progressively broken down into individual constituents. The subject of interest in this study is the behaviour of concrete at the mesoscale. At the mesoscale, concrete can be thought of as a multi-phased material that comprises of the cement paste (mortar) surrounding the coarse and the fine aggregates leading to a highly heterogeneous structure. The heterogeneity of concrete and the forces binding its various phases are normally overlooked when analysing concrete at the macroscopic level. The simplifying assumption of concrete as a uniform homogeneous material neglects many of the key aspects such as the aggregate shape and the size distribution which play a significant

role in determining its macroscopic strength and fracture behaviour. These aspects can be better studied when concrete is analysed at the mesoscale.

The motivation to further comprehend the complex damage initiation of microcracks, their progressive development into major cracks leading to failure of concrete and how the morphology and the content and/or distribution of the different phases of the concrete at the mesoscale relate to the physical properties observed at the macroscale mechanical responses has seen numerous effort devoted towards the modelling of concrete at the mesoscale e.g. $[3,50,54,48,32,16]$. These approaches can be identified by the manner in which the heterogeneity of concrete at the mesoscale is represented. The first approach adopts an implicit representation of the different phases of concrete at smaller length scales through a description of stochastic fields of material properties affecting the behaviour of concrete within the computational domain $[4,55,54]$. The second approach explicitly discretises the various phases present in the concrete mesostructure. The behaviour of the concrete is then 
modelled using a suitable computational procedure. The explicit approach will be the focus of this study, particularly on the computational procedure that is being adopted. Many types of computational models have been reported in the literature. These include lattice models [36, 35, 13], discrete element models [27, 25, 45] and continuum models [51, 16, 32].

The lattice models involve the use of structural finite elements e.g. beams and truss to form a lattice network to represent the concrete mesostructure. The heterogeneity of concrete is modelled by assigning different material properties to the beam elements (mortar or aggregate) depending on the elements' positions with reference to the concrete mesostructure. Lattice models have been successfully applied to model the failure of concrete at the mesoscale for various types of loadings under both two- and three-dimensional conditions [35, 19, 38, 10, 37]. Fracture is modelled through various approaches e.g. removing elements that exceed the tensile strength of concrete [35], particle lattice models [7] and flexible nodes [10]. Lattice models offer computational simplicity due to the use of simple beam theories for the underlying lattice whereas its disadvantages include the need of a very fine mesh to accurately represent the morphology of the concrete mesostructure and to avoid mesh bias in the solutions.

The discrete element method models the behaviour of materials through the use of discs and spheres [6]; and polygonal particles [11]. The force transfer between the particles is achieved through contact interaction. The motion of the particles is resolved using rigid body dynamics. To model the heterogeneity of concrete, each discrete element is assigned different material properties depending on whether they belong to the aggregate or mortar phase, similar to the lattice models. Inter-particle spring elements are used to model the cohesion between the various phases in the mesostructure. The spring elements enable separation between particles when the internal force in the spring exceeds the bond strength. In this way, fracture within a meso-scale structure can be modelled. The application of the discrete element method to model concrete fracture at the mesoscale has been reported in many instances e.g. [27, 25, 45]. Similar to the lattice models, the advantage of discrete element models lie in their simplicity but incur a penalty in the required size of the computational model. Moreover, the discrete element models require extensive calibration of the bond strength and inter-particle contact stiffness prior to numerical simulations.

On another front, continuum models are generally based on the finite element method (FEM) although investigations based on peridynamics has also been reported [53]. The entire concrete meso-structure (aggregate and mortar) is explicitly discretised using standard finite elements with different material properties assigned to the different phases in the computational domain. The fracture in the meso-structure can be explicitly modelled using interface elements e.g. [23, 40, 48, 32, 16] or implicitly modelled using damage models e.g. 
[38, 39, 2, 47] or enrichment functions via the extended FEM e.g. [9].

Interface elements model the fracture of the concrete mesostructure through nonlinear traction-separation laws that characterise the bond between the mortar and the aggregate phases. The interface elements connect the continuum elements and are pre-inserted into the finite element mesh prior to the numerical simulations. As a consequence, fracture is allowed only along the element interfaces. Different types of interface elements have been formulated including zero-thickness quadrilateral elements [48] in two dimensions, prismatic elements (6-nodes or 8-nodes) in three dimensions [43,44], and continuum elements with high aspect ratio formulated from the kinematics of the continuum strong discontinuity approach [32]. When damage models are employed, fracture is modelled within the continuum elements. A damage variable associated with the degradation of the material that acts as a stiffness reduction factor when microcracks and voids coalesce and propagate is defined to model the failure process in the mesostructure. The challenge in the application of damage models involve the modelling of the very thin region at the interface between the matrix and the aggregate. A high mesh density is usually required in the vicinity of these regions [24]. In the extended FEM, the fracture in concrete is defined through nodes that are enriched with a Heaviside function to describe the discontinuity between the crack faces. Traction-separation laws are defined across the elements enriched by the Heaviside function to model the cohesion along the interfaces [33].

This study describes a continuum approach coupled with zero-thickness cohesive interface elements to model mesoscale fracture of concrete. The scaled boundary finite element method (SBFEM) [42] is used to discretise the aggregates and the mortar phases in the concrete mesostructure. A mesh fragmentation process is then applied so that zero-thickness interface elements can be inserted along the cell boundaries. This approach differs from that developed by Huang et al. [17] and Wang et al. [49] in which a single SBFEM is employed to discretise the aggregates whereas the mortar is discretised with standard finite elements [17] or polygonal scaled boundary finite elements [49]. Instead, a quadtree decomposition in combination with polygon meshes within the framework of the SBFEM [26] is adopted. Quadtree meshes are particularly suitable for the implementation with the SBFEM, because the presence of hanging nodes do not impose any restriction on the SBFEM. Each cell is modelled as if it were an arbitrary sided polygon similar to the approach of Tabarraei and Sukumar [46]. The flexibility to using arbitrary sided polygons also enables accurate representation of the problem geometry at the interfaces between the aggregate and the matrix.

The application of quadtree decomposition leads to an efficient approach for mesh generation from digital images of concrete mesostructures [56, 21, 34]. Due to its ability to discretise the geometric features of different scales efficiently, quadtree meshes avoid the generation 
of very fine meshes when compared with pixel-based approaches e.g. [15, 31]. Moreover, the whole image-based mesh generation process is fully automatic and significantly reduces the time and human effort required to convert an image to a mesh suitable for analysis. Quadtree meshes also facilitate efficient computations due to the limited patterns of cells in the mesh. These can be pre-computed, stored in the memory and quickly extracted when required. This significantly limits the required number of additional computations to only the polygon shaped cells situated along the boundary between two dissimilar materials.

This paper is organised as follows: Section 2 describes the procedure in which digital images are used for mesh generation using a balanced quadtree decomposition and a smoothing operation to better discretise the boundaries of aggregates in the mesh. Section 3 reviews the fundamental of the SBFEM used to model the aggregate and mortar phases of the concrete mesostructure. The zero-thickness interface elements required to model the fracture within the concrete mesostructure are described in Section 4. Parametric studies are carried out in Section 5 to investigate the effect of various parameters at the mesoscale e.g. aggregate-to-weight ratio, aggregate size distribution on the strength of concrete. Qualitative comparisons with results reported in the literature will be made. The feasibility of the developed approach to model mesoscale fracture of concrete specimens obtained from X-ray computed tomography (XCT) is also demonstrated. The major conclusions of the study are summarised in Section 6.

\section{Hybrid Quadtree Image-based Mesh Generation}

Digital images of real or representative concrete mesostructures are used as inputs for the mesoscale fracture analyses. The images may be obtained from XCT scans e.g. [31]. Al-

ternatively, representative concrete mesostructures can be generated from a take-and-place algorithm e.g [51, 32]. In this study, digital images obtained from both the aforementioned techniques will be used. Computational meshes are generated directly from the digital images using quadtree decompositions. The use of quadtree meshes in mesh generation is complementary to the SBFEM. Each cell is modelled as an arbitrary sided polygon irrespective of the presence of hanging nodes. The following sections describe the mesh generation process from digital images.

\subsection{Quadtree mesh generation}

Quadtree mesh generation involves the recursive subdivision of a space into four quadrants or regions of equal dimensions. Each quadrant can be further partitioned into four smaller 


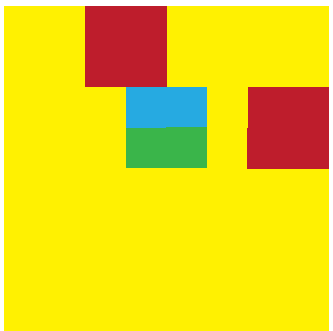

(a) digital image

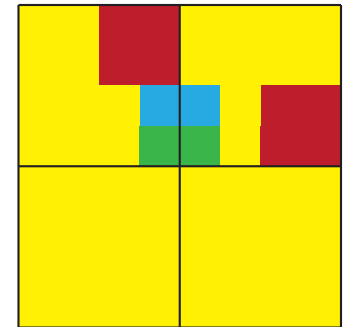

(b) initial decomposition

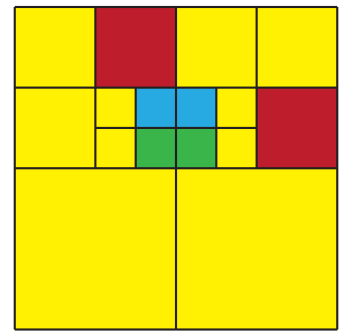

(c) quadtree mesh

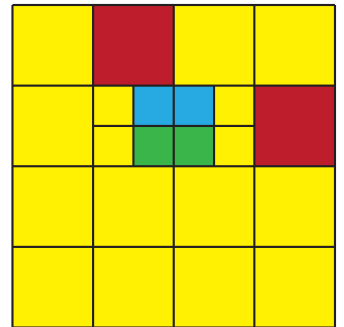

(d) balanced quadtree mesh

Figure 1: Balanced quadtree mesh generation.

quadrants referred to as "children" if a higher resolution is required in a desired region. The information of each region is stored in a hierarchical data structure likened to a tree. Between two adjacent levels, the quadrant at the top of the tree is the coarser "parent", which becomes more refined according to prescribed parameters further along the hierarchy. MATLAB provides a built-in function 'qtdecomp' which performs quadtreee decomposition of image files based on the homogeneity of each divided region i.e. there can be only one colour within a quadrant. If a divided region meets the homogeneity criterion it will not be divided any further. Conversely, if the criterion is not met, the region is further divided into four smaller square regions. A subsequent homogeneity test is applied to the new quadrants that are generated from the quadtree decomposition. This process is repeated until all the cells generated from the image satisfy the homogeneity criterion.

Fig. 1a shows a generic image which is to be discretised with a quadtree mesh. The homogeneity criterion used here is the color of each cell. An initial application of the quadtree decomposition will split the image in Fig. 1a into four cells as shown in Fig. 1b. At this juncture, the two cells at the bottom satisfy the homogeneity criterion and will not be further partitioned. The two cells on the top does not satisfy the homogeneity criterion. They are further partitioned until the homogeneity criterion is satisfied in all the cells as shown in Fig. 1c.

Depending on the information of an input image, certain regions may require further refinement in order to satisfy the homogeneity criterion compared with other regions. This can potentially create an unbalanced mesh resulting in large size differences between adjacent cells where the quadtree transitions from coarse to fine regions. Referring to Fig. 1c, examples of such cells are the large ones at the bottom of the mesh. It is therefore common practice to impose restrictions on the maximum size difference between adjacent cells. This is referred to as a balancing condition, which results in the final mesh being considered balanced or restricted, once applied. A commonly imposed balancing condition is the 2:1 rule which 


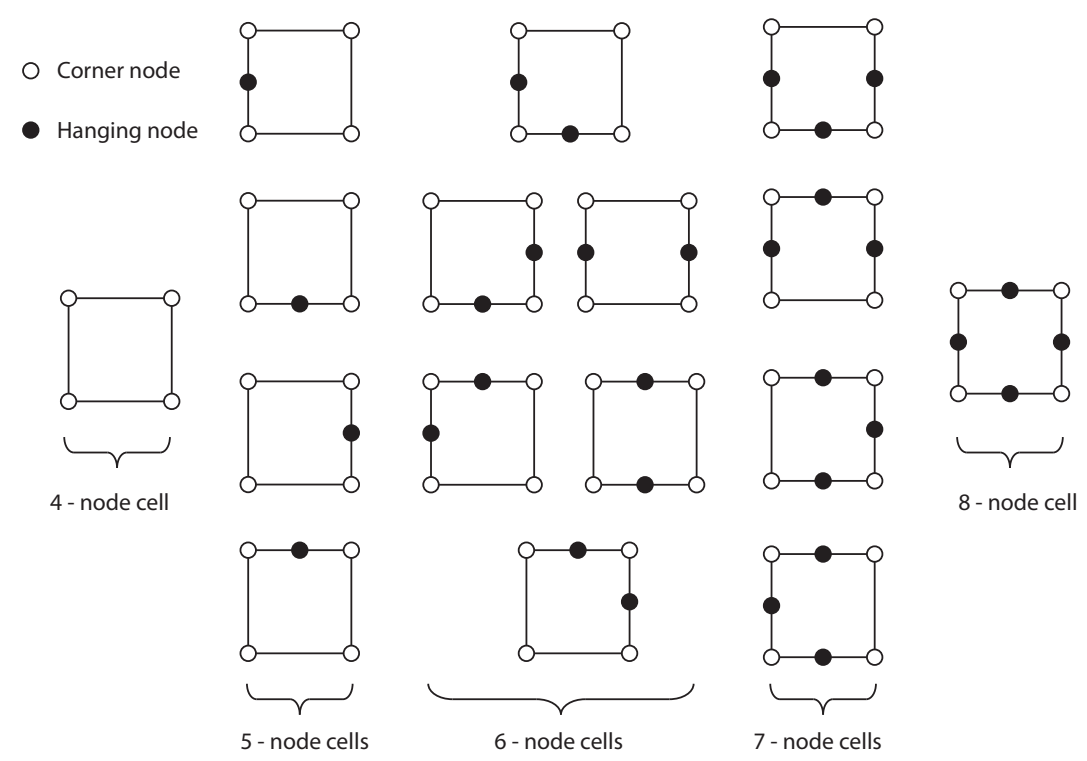

Figure 2: Quadtree cells belonging to a balanced quadtree decomposition.

requires no node/region in the quadtree to be adjacent to one less than half its size [30]. For the mesh in Fig. 1c, this is achieved by further partitioning the large cells at the bottom of the mesh into four quadrants resulting in the balanced quadtree mesh in Fig.1d.

When a balanced quadtree mesh is adopted, the number of types of cells in the mesh is limited to only the types shown in Fig. 2. This can be exploited for efficient computations in large scale computations or moving boundary problems [46, 26]. Only the information of the types of cells in a balanced quadtree mesh are required and these can be pre-computed, stored in the CPU memory and retrieved as needed during the actual computations.

\subsection{Boundary smoothing along material interfaces}

A purely quadtree decomposition will result in a mesh composed entirely of squares with only horizontal and vertically aligned sides. For this reason, the interface between the aggregates and the matrix in the concrete meso-structure cannot be realistically discretised using a purely quadtree decomposition unless these regions are sufficiently refined. Unrealistically high stresses can develop along the artificially induced jagged edges on the interfaces of different materials [22, 34]. Although this effect can be reduced with a high level of mesh refinement, discretisation error caused by the artificially induced jagged edges cannot be entirely eliminated even with overly refined discretisations in these regions.

One alternative to resolve the artificial discretisation of the curved and irregular features is to adopt a hybrid quadtree-polygon mesh [26]. Within this framework, the bulk of the mesh is comprised of the 16 types of quadtree cells shown in Fig. 2. Along dissimilar material 


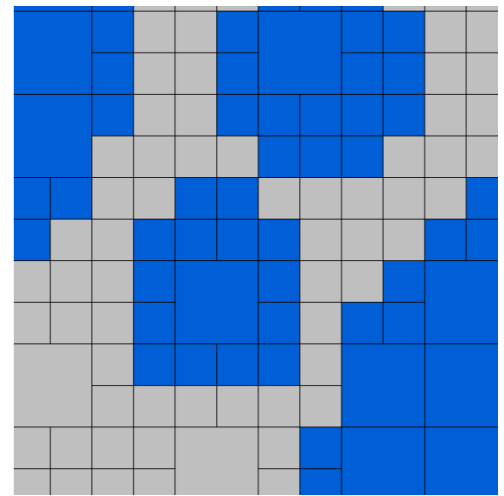

(a) balanced quadtree decomposition

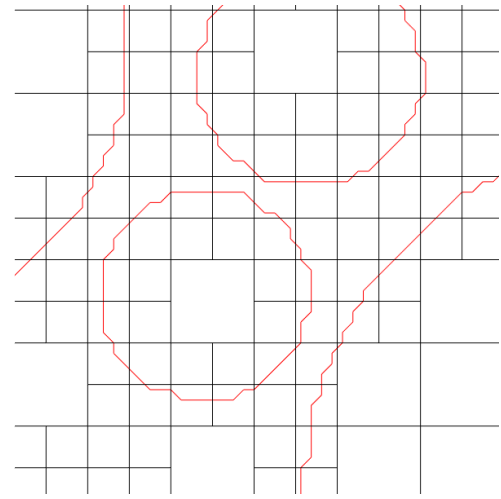

(b) image contour detection

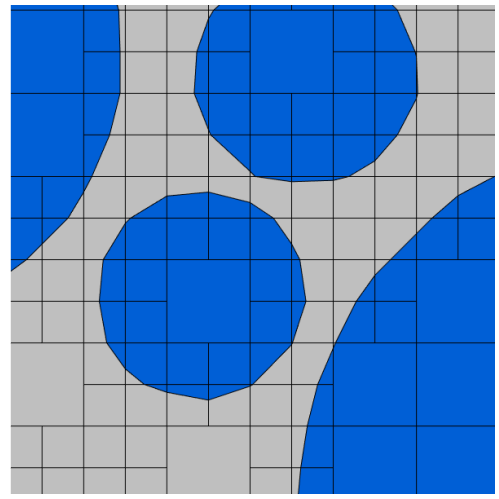

(c) spline smoothing

Figure 3: Spline smoothing at material interfaces.

interfaces and the irregular boundaries of the domain, arbitrary sided polygons are adopted for a more realistic discretisation. This approach is adopted in this study and its application to a generic image is shown in Fig. 3.

A boundary smoothing process is applied on the balanced quadtree decomposition (Fig. 3a) of an image after it has been generated. MATLAB's built-in function 'contour' is adopted to identify the boundaries and the material interfaces in the concrete meso-structure (Fig. 3b). The locations at which these contours intersect each quadrant in the quadtree decomposition can be found from the change in the contour heights computed from the pixel colours of the image. Once these intersections have been identified, a smoothing procedure is applied to adjust the segmented contours and align them to form the smoothed boundaries of the image. MATLAB's built-in function 'smooth', which adopts a 5-point moving average technique is used for this purpose. The smoothed contours determine the new boundaries of the material interfaces in the mesh. Their intersections with the edges of the quadtree cells can be used to cut these cells leading to two arbitrary sided polygons, which are naturally modelled by the SBFEM.

Although the smoothing process increases the number of different cell types in the final mesh, representation of the boundaries of dissimilar materials is more realistic (see Fig. 3c). The artificial jagged edges along dissimilar materials of a purely quadtree decomposition (see Fig. 3a) are eliminated with spline smoothing. The discretisation along the boundary is also coarser as compared with a purely quadtree discretisation employing a high mesh refinement at the material interfaces. This is significant in reducing the the overall number of degrees-of-freedom of the mesh. Moreover, the polygonal cells are only present along the boundaries between the aggregate and mortar phases. 


\section{Scaled Boundary Finite Element Formulation}

The SBFEM can be formulated on polygons with an arbitrary number of sides so as long as the polygon geometry satisfies a scaling requirement. This requires the definition of a point, $\left(x_{0}, y_{0}\right)$, called the scaling centre in the polygon from which the entire boundary is visible. With reference to the definition of the scaling requirement, convex polygons, which include any type of quadtree cells can be modelled by the SBFEM. A generic polygonal cell modelled by the SBFEM is shown in Fig. 4a. The position of a point in the SBFEM is described by a radial-circumferential like coordinate system $(\xi, \eta)$ defined over a triangular sector bounded by the scaling centre with coordinates and a line element as shown in Fig. 4b. The radial coordinate $\xi$ is defined from $\left(x_{0}, y_{0}\right)$ to a point on the line element and has a range $0 \leq \xi \leq 1$ with $\xi=0$ at the scaling centre and $\xi=1$ at the line element. The local coordinate $\eta$ is defined similar to the FEM and has a range $-1 \leq \eta \leq 1$. Several triangular sectors are combined to form a closed loop polygon. The Cartesian coordinates $(x, y)$ of a point bounded by the triangular sector shown in Fig. $4 \mathrm{~b}$ is related to the SBFEM coordinates by

$$
\left\{\begin{array}{l}
x(\xi, \eta) \\
y(\xi, \eta)
\end{array}\right\}=\left\{\begin{array}{l}
x_{0} \\
y_{0}
\end{array}\right\}+\xi \mathbf{N}_{u}(\eta) \mathbf{x}_{b}
$$

where $\mathbf{x}_{b}=\left[\begin{array}{lllll}x_{1} & y_{1} & x_{2} & y_{2} & \ldots\end{array}\right]^{T}$ is the nodal coordinate vector of the line elements on the boundary with $\left(x_{i}, y_{i}\right)$ as nodal coordinates and $\mathbf{N}_{u}(\eta)$ is the shape function matrix. In this study, linear shape functions are used. Therefore $\mathbf{N}_{u}(\eta)$ has the form

$$
\mathbf{N}_{u}(\eta)=\left[\begin{array}{cccc}
N_{1}(\eta) & 0 & N_{2}(\eta) & 0 \\
0 & N_{1}(\eta) & 0 & N_{2}(\eta)
\end{array}\right]
$$

and $N_{i}(\eta)$ are standard one-dimensional finite element linear shape functions.

Within a triangular sector, the displacement field $\mathbf{u}(\xi, \eta)$ is defined as

$$
\mathbf{u}(\xi, \eta)=\mathbf{N}_{u}(\eta) \mathbf{u}_{h}(\xi)
$$

where $\mathbf{u}_{h}(\xi)$ are analytical displacement functions obtained from the solution of the equilibrium condition within a cell. The equilibrium condition in a polygon can be formulated from the principle of virtual work [8] or the method of weighted residuals [42]. This results in the scaled boundary finite element equation in displacement

$$
\mathbf{E}_{0} \xi^{2} \mathbf{u}_{h}(\xi)_{, \xi \xi}+\left(\mathbf{E}_{0}+\mathbf{E}_{1}^{T}-\mathbf{E}_{1}\right) \xi \mathbf{u}_{h}(\xi)_{, \xi}-\mathbf{E}_{2} \mathbf{u}_{h}(\xi)=0
$$




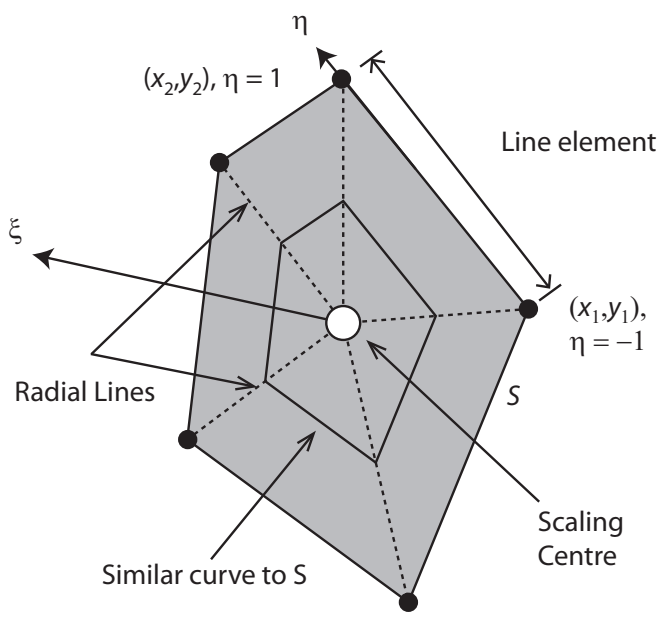

(a) generic polygon

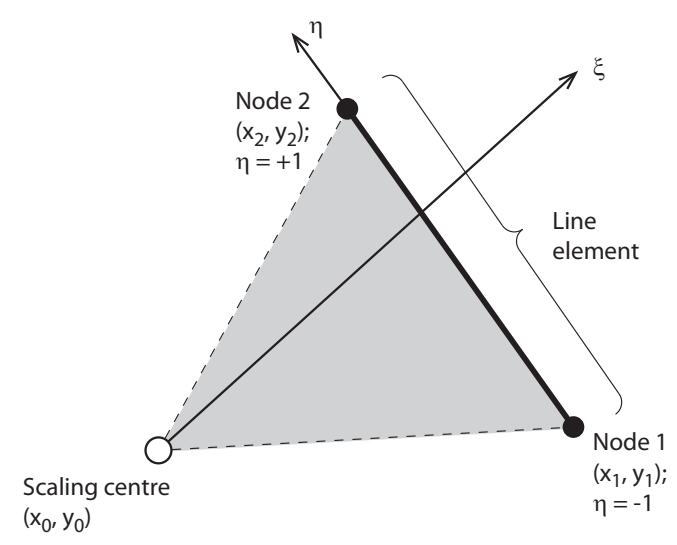

(b) a triangular sector forming part of a polygon

Figure 4: Scaled boundary finite element discretisation of a generic polygon.

where $\mathbf{u}_{h}(\xi)_{, \xi}$ and $\mathbf{u}_{h}(\xi)_{, \xi \xi}$ are the first and second derivatives of $\mathbf{u}_{h}(\xi)$ with respect to $\xi$, respectively. The coefficient matrices $\mathbf{E}_{i}, i=0,1$ and 2 are defined as

$$
\begin{aligned}
& \mathbf{E}_{0}=\int_{-1}^{1} \mathbf{B}_{1}^{T}(\eta) \mathbf{D B}_{1}(\eta) J d \eta \\
& \mathbf{E}_{1}=\int_{-1}^{1} \mathbf{B}_{2}^{T}(\eta) \mathbf{D B}_{1}(\eta) J d \eta \\
& \mathbf{E}_{2}=\int_{-1}^{1} \mathbf{B}_{2}^{T}(\eta) \mathbf{D B}_{2}(\eta) J d \eta
\end{aligned}
$$

In Eq. (5) - Eq. (7), $\mathbf{B}_{1}(\eta)$ and $\mathbf{B}_{2}(\eta)$ are the SBFEM strain-displacement matrices, $\mathbf{D}$ is the material constitutive matrix and $J$ is the determinant of the Jacobian matrix $\mathbf{J}(\eta)$ required for coordinate transformation [41]

$$
\mathbf{J}(\eta)=\left[\begin{array}{cc}
x_{\eta} & y_{\eta} \\
x_{\eta, \eta} & y_{\eta, \eta}
\end{array}\right]
$$

where

$$
\left[\begin{array}{ll}
x_{\eta} & y_{\eta}
\end{array}\right]^{T}=\mathbf{N}_{u}(\eta) \mathbf{x}_{b}
$$

and $x_{\eta, \eta}$ and $y_{\eta, \eta}$ are the first derivatives of $x_{\eta}$ and $y_{\eta}$ with respect to $\eta$. The coefficient matrices $\mathbf{E}_{i}$ are assembled over all the triangular sectors in a cell in a manner similar to the assembly process of the FEM. 
The solution of the second order differential equation in Eq. (4) for a cell is obtained by first transforming it into a first order differential equation with twice the number of unknowns as [41]

$$
\xi\left\{\begin{array}{l}
\mathbf{u}_{h}(\xi) \\
\mathbf{q}_{h}(\xi)
\end{array}\right\}_{, \xi}=-\mathbf{Z}\left\{\begin{array}{l}
\mathbf{u}_{h}(\xi) \\
\mathbf{q}_{h}(\xi)
\end{array}\right\}
$$

where $\mathbf{q}_{h}(\xi)$ is a vector of analytical functions related to the internal forces

$$
\mathbf{q}_{h}(\xi)=\mathbf{E}_{0} \mathbf{u}_{h}(\xi), \xi+\mathbf{E}_{1}^{T} \mathbf{u}_{h}(\xi)
$$

and $\mathbf{Z}$ is a Hamiltonian matrix

$$
\mathbf{Z}=\left[\begin{array}{cc}
\mathbf{E}_{0}^{-1} \mathbf{E}_{1}^{T} & -\mathbf{E}_{0}^{-1} \\
-\mathbf{E}_{2}+\mathbf{E}_{1} \mathbf{E}_{0}^{-1} \mathbf{E}_{1}^{T} & -\mathbf{E}_{1} \mathbf{E}_{0}^{-1}
\end{array}\right]
$$

Eq. (10) is solved by decomposing $\mathbf{Z}$ into base vectors. A Schur decomposition of $\mathbf{Z}$ is performed. This results in

$$
\mathrm{ZV}=\mathrm{VS}
$$

The Schur matrix $\mathbf{S}$ has the following form

$$
\mathbf{S}=\left[\begin{array}{cc}
\mathbf{S}_{n} & * \\
& \mathbf{S}_{p}
\end{array}\right]
$$

where $\mathbf{S}_{n}$ and $\mathbf{S}_{p}$ are upper triangular matrices with negative and positive eigenvalues of $\mathbf{Z}$ along their diagonals. The transformation matrix $\mathbf{V}$ can be partitioned as

$$
\mathbf{V}=\left[\begin{array}{ll}
\mathbf{V}_{u} & \overline{\mathbf{V}}_{u} \\
\mathbf{V}_{q} & \overline{\mathbf{V}}_{q}
\end{array}\right]
$$

The matrices $\mathbf{V}_{u}$ and $\overline{\mathbf{V}}_{u}$ are related to the displacement modes of a cell whereas the matrices $\mathbf{V}_{q}$ and $\overline{\mathbf{V}}_{q}$ are related to the modal forces. For bounded domains such as the quadtree and polygonal cells considered in this study, only the Schur matrix containing negative eigenvalues $\mathbf{S}_{n}$ and its corresponding modal displacements $\mathbf{V}_{u}$ and modal forces $\mathbf{V}_{q}$ lead to finite displacements at the scaling centre. The solution for the analytical displacement 
functions $\mathbf{u}_{h}(\xi)$ and analytical internal force functions can then be expressed as

$$
\begin{aligned}
& \mathbf{u}_{h}(\xi)=\mathbf{V}_{u} \xi^{-\mathbf{S}_{n}} \mathbf{c} \\
& \mathbf{q}_{h}(\xi)=\mathbf{V}_{q} \xi^{-\mathbf{S}_{n}} \mathbf{c}
\end{aligned}
$$

where $\mathbf{c}$ are the integration constants that depend on the boundary conditions and can be computed from the nodal displacements in each polygon as

$$
\mathbf{c}=\mathbf{V}_{u}^{-1} \mathbf{u}_{b}
$$

and $\mathbf{u}_{b}$ is the polygon nodal displacement vector. Substituting Eq. (16) into Eq. (3), the complete displacement field in a triangular sector bounded by the scaling centre and a line element on the boundary of a polygon can be expressed as

$$
\mathbf{u}(\xi, \eta)=\mathbf{N}_{u}(\eta) \mathbf{V}_{u} \xi^{-\mathbf{S}_{n}} \mathbf{c}
$$

The stiffness matrix of a cell $\mathbf{K}_{\text {cell }}$ is defined in Eq. (15) as

$$
\mathbf{K}_{\text {cell }}=\mathbf{V}_{q} \mathbf{V}_{u}^{-1}
$$

The corresponding strain field $\boldsymbol{\epsilon}(\xi, \eta)$ is formulated from its definition and the use of the SBFEM coordinate transformation as [41]

$$
\boldsymbol{\epsilon}(\xi, \eta)=\mathbf{B}_{1}(\eta) \mathbf{u}_{h}(\xi)_{, \xi}+\xi^{-1} \mathbf{B}_{2}(\eta) \mathbf{u}_{h}(\xi)
$$

Substituting Eq. (16) into Eq. (21), the strain can be expressed as

$$
\boldsymbol{\epsilon}(\xi, \eta)=\boldsymbol{\Psi}_{\epsilon}(\eta) \xi^{-\mathbf{S}_{n}-\mathbf{I}} \mathbf{c}
$$

where $\mathbf{I}$ is an identity matrix and the strain mode $\boldsymbol{\Psi}_{\epsilon}(\eta)$ is defined as

$$
\boldsymbol{\Psi}_{\epsilon}(\eta)=-\mathbf{B}_{1}(\eta) \mathbf{V}_{u} \mathbf{S}_{n}+\mathbf{B}_{2}(\eta) \mathbf{V}_{u}
$$

If the cells behave in a linear elastic manner the stress field $\boldsymbol{\sigma}(\xi, \eta)$ is expressed using the Hooke's law as

$$
\boldsymbol{\sigma}(\xi, \eta)=\mathbf{D} \boldsymbol{\epsilon}(\xi, \eta)
$$

The flexibility to formulate the SBFEM over arbitrary sided polygons makes it particularly 
suitable for implementation with quadtree meshes. Within this framework, each cell in the mesh is treated as an arbitrary sided polygon modelled by the SBFEM. A similar framework was previously adopted by Tabarraei and Sukumar [46] for $h$-adaptive computations with the FEM, where instead of the SBFEM, polygonal interpolants based on the natural neighbour shape functions are used. In addition to the advantages that can be gained in automatic mesh generation (as was previously outlined in Section 2), quadtree meshes also facilitate more efficient computations particularly when a balanced quadtree mesh is employed. The geometric restrictions of balanced quadtree meshes limit the number of quadtree cells to only the sixteen types shown in Fig. 2. These cells are referred to as master cells [26]. For homogeneous, isotropic linear elastic materials, the stiffness and mass matrices of these cells can be pre-calculated before the analysis and stored within the CPU memory. During the analysis, they can be quickly retrieved (and scaled in a dynamic analysis) for quick computations. Additional computations of the stiffness matrix are required only for the arbitrary sided polygonal cells that model the boundaries of the aggregates and mortar phases.

\section{Meso-scale Cohesive Fracture Representation}

To model the fracture at the mesoscale, the hybrid quadtree-polygon mesh is further augmented to allow insertion of interface elements along the boundaries of each cell. This process involves first, fragmentation of an initial mesh such as that shown in Fig. 5a by duplicating the nodes and edges that are shared by two (or more) cells in the mesh. The number of nodes to be duplicated depends on the connectivity of the node to the cells. For a node that is shared by $n$ number of cells, the number of duplicate nodes is $n-1$. At the same time, a common edge shared by adjacent cells is also duplicated. The cell-nodal connectivity is then regenerated taking into account the new nodes and edges generated by the fragmentation process. This results in the mesh shown in Fig. 5b where the duplicated nodes are shown in italics. Each cell is now self-contained and does not share a common node with its adjacent cells.

The cells in the mesh are then reconnected again using zero-thickness 4-node cohesive interface elements (Fig. 6) developed by Gerstle and Xie [12] along all the internal cell boundaries that previously share a common edge with an adjacent cell/polygon. This results in the mesh shown in Fig. 5c. The mesh fragmentation process adopted in this study resembles those adopted in the FEM e.g. [52, 54, 48, 31]. Instead of standard finite element shapes, quadtree and polygonal meshes are adopted in this study.

The cohesive interface elements are used to model all potential crack paths in the con- 


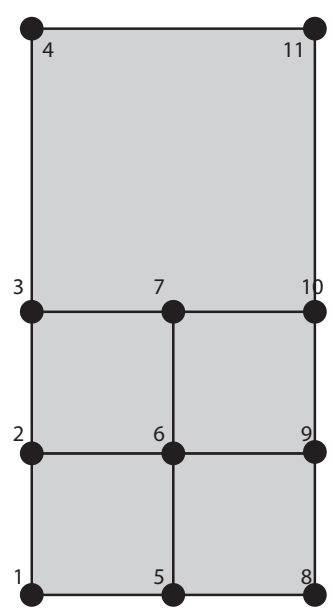

(a) initial quadtree mesh

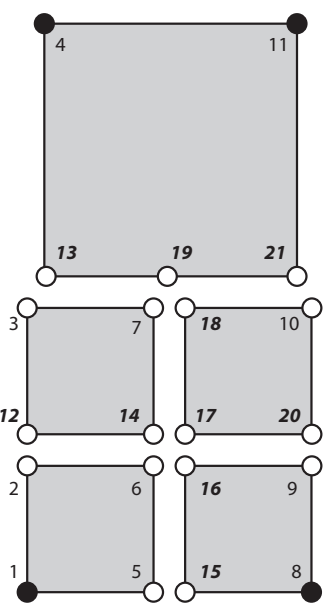

(b) splitting nodes and edges

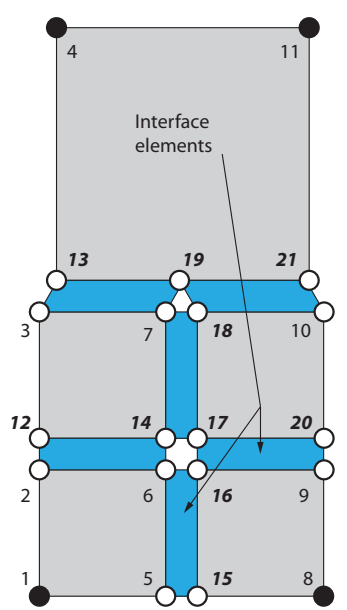

(c) inserting interface elements

Figure 5: Pre-inserting interface elements into balanced quadtree mesh.

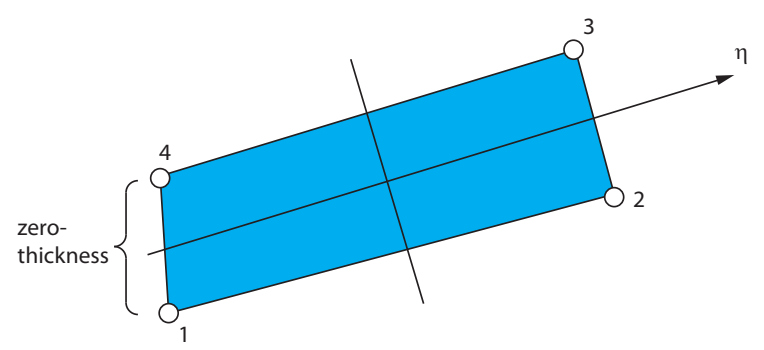

Figure 6: Zero-thickness interface elements. 
crete mesostructure along three possible interfaces: aggregate-mortar; mortar-motar; and aggregate-aggregate in the mesh. Fracture is allowed to propagate only along the boundaries of the cells in the mesh. The interface elements have zero-thickness and are characterised by traction-separation laws in both normal and tangential directions. Different types of traction-separation laws can be defined e.g. linear softening (Fig. 7) or bilinear softening as widely used in Yang et al. [54], Huang and Li [15], Wang et al. [48], Ren et al. [31]. The constitutive relation between the normal and tangential stress with the relative normal and tangential displacement between two opposing edges of an interface element is

$$
\left\{\begin{array}{c}
\tau \\
\sigma
\end{array}\right\}=\mathbf{C}\left\{\begin{array}{c}
s \\
w
\end{array}\right\}
$$

where $\sigma$ and $\tau$ are the normal and tangential stress along the element and $w$ and $s$ are the relative opening and sliding displacements, respectively. The matrix $\mathbf{C}$ is defined as

$$
\mathbf{C}=\left[\begin{array}{ll}
k_{s} & k_{s n} \\
k_{s n} & k_{n}
\end{array}\right]
$$

where $k_{n}$ and $k_{s}$ are the secant stiffnesses of the traction-separation curves in the normal and tangential directions, respectively. The variable $k_{s n}$ in Eq. (26) represents the additional stiffness due to the interaction between the shear and the normal fracture mechanisms. It is not considered in this study as the magnitude of the normal shear interaction is not reported in the literature. Therefore, the proposed method will be subjected to situations in which there might be shear reaction to the tangential relative displacement (slip) even when the crack is completely opened. It is also to note that the approach adopted in this study should have no restriction on the type of constitutive model that can be implemented with the SBFEM. More complex constitutive models can be considered if desired. For example, implementation with a constitutive formulation that conforms to work-softening elasto-plasticity e.g. [23] may also be attempted. The magnitudes of tensile strength $f_{t}$, critical opening displacement $w_{c}$, shear strength $f_{s}$ and critical sliding displacement $s_{c}$ that define the traction-separation laws depend on whether the interface elements are connected to the mortar-mortar phase, the aggregate-mortar phase or the aggregate-aggregate phase.

The stiffness matrix of each interface element is computed as

$$
\mathbf{K}_{i t z}=\int \mathbf{N}_{z}^{T}(\eta) \mathbf{C N}_{z}(\eta) d \eta
$$




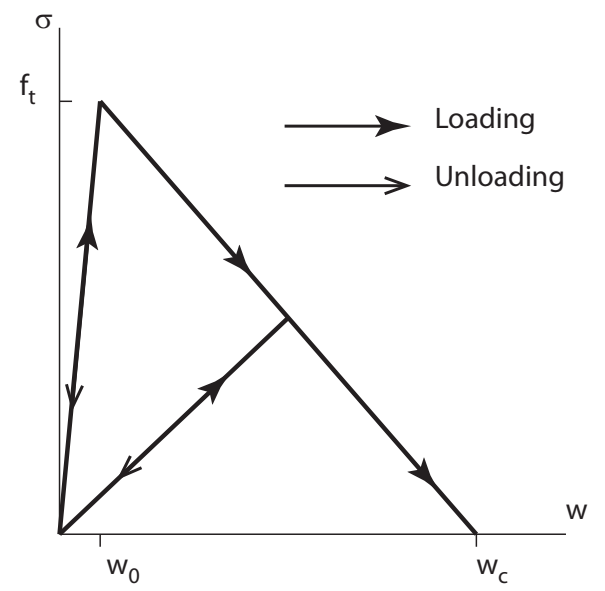

(a) normal

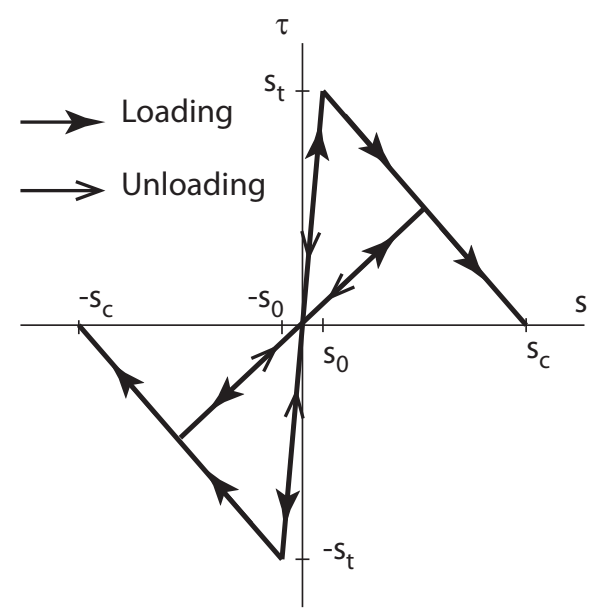

(b) tangential

Figure 7: Generic traction separation curves for fracture modelling.

where the shape function matrix $\mathbf{N}_{z}(\eta)$ is defined as

$$
\mathbf{N}_{z}(\eta)=\left[\begin{array}{lllllll}
-N_{1}(\eta) & & -N_{2}(\eta) & & N_{2}(\eta) & & N_{1}(\eta) \\
& -N_{1}(\eta) & & -N_{2}(\eta) & & N_{2}(\eta) & \\
& & N_{1}(\eta)
\end{array}\right]
$$

The shape functions $N_{1}(\eta)$ and $N_{2}(\eta)$ are standard one-dimensional linear finite element interpolants. Standard Gaussian quadrature can be employed to integrate $\mathbf{K}_{i t z}$. The interface elements are compatible with the SBFEM formulation and can be coupled directly without any special techniques. The compatibility arises due to the use of linear shape functions along the quadtree and polygonal cells and along the edges of the interface elements in the mesh. The stiffness matrix in each interface element can be assembled with $\mathbf{K}_{\text {cell }}$ similar to the assembly process in the FEM.

\section{Mesoscale Fracture Modelling}

\subsection{Parametric Studies}

Parametric studies are carried out to determine the influence of mesostructure heterogeneity e.g. aggregate to volume ratio, aggregate size and specimen porosity on the fracture characteristics of concrete. For this purpose, a take-and-place algorithm is implemented to generate specimens representative of concrete mesostructure.

The mesostructure of a specimen is generated using a Java programming environment called Processing [29]. A take-and-place algorithm is adapted from the work of Wriggers and 


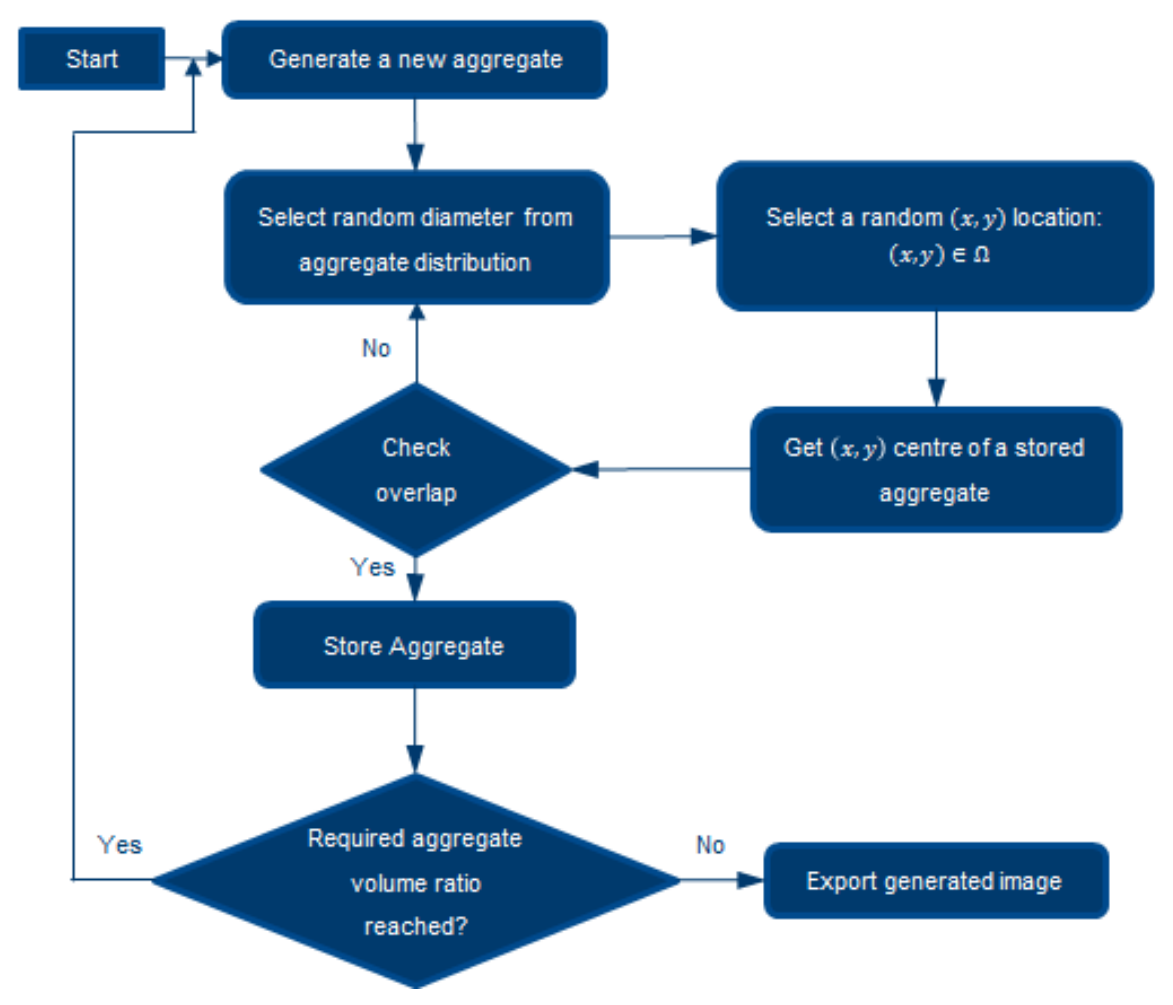

Figure 8: Flow diagram of aggregate placing algorithm.

Moftah [51]. Only the intervals of the aggregate diameters in a sample are required as input, which can be determined based on the sieve size and the specimen's aggregate volume ratio. The size distribution of the aggregates in a concrete mesostructure is first determined using Fuller's grading curve

$$
P(d)=100\left(\frac{d}{d_{\max }}\right)^{n}
$$

where $P(d)$ is the cumulative percentage of an aggregate to pass a sieve with diameter $d, d_{\max }$ is the maximum aggregate size and $n$ is an exponent defined in the range $0.45 \leq n \leq 0.70$. The area of the aggregates within an interval $\left[d_{i}, d_{i+1}\right]$ is then computed as [51]

$$
A_{p}\left[d_{i}, d_{i+1}\right]=\frac{P\left(d_{i}\right)-P\left(d_{i+1}\right)}{P\left(d_{\max }\right)-P\left(d_{\min }\right)} \mu_{p}
$$

where $\mu_{p}$ is the volume fraction of the mesostructure, $A$ is the total area of the specimen, $A_{p}\left[d_{i}, d_{i+1}\right]$ is the volume fraction of the aggregates defined within $\left[d_{i}, d_{i+1}\right]$ and $d$ is the size of the sieve.

The process of aggregate generation in a generic mesostructure is outlined in Fig. 8. The aggregates are assumed to be circular with diameter $d$. The algorithm considers only 
the placement of coarse aggregates i.e. aggregates with diameters $d \geq 4.75 \mathrm{~mm}$. Fine aggregates, which comprise of sand and crushed stone are assumed to belong to the mortar phase of concrete. The algorithm requires as inputs, the size of the concrete mesostructure, the aggregate volume ratio $\mu_{p}$ and the range of the aggregate diameters expected in the sample $\left[d_{1}, d_{2}, d_{3} \ldots, d_{n}\right]$.

Starting from a random value of diameter sampled within the range $\left[d_{i}, d_{i+1}\right]$, the algorithm continuously places the centre of the aggregate at random locations within the domain of the mesostructure $\Omega$. The position of the aggregate is checked to ensure that it neither overlaps other previously generated aggregates nor placed outside the bounding box defining the boundary of the mesostructure. The aggregates are assumed to be coated around with a mortar file having a certain minimum thickness $\gamma d$ similar to the approach of Wriggers and Moftah [51]. In this study, $\gamma=0.02$ is adopted.

Once a suitable size and location of an aggregate is found, the algorithm stores the aggregate in an object array list and breaks out of the loop. This process is then repeated for each of the interval $\left[d_{i}, d_{i+1}\right]$ until the domain of the mesostructure reaches the target $\mu_{p}$. The draw function in Processing is then executed to generate the image before saving in a desired format.

Concrete specimens with dimensions $100 \mathrm{~mm} \times 100 \mathrm{~mm}$ and subjected to uniaxial tension as shown in Fig. 9 are considered. Plane stress conditions are assumed. The specimens have an out of plane thickness of $150 \mathrm{~mm}$. The right edge of a specimen is subjected to a uniform horizontal displacement $u$ and the left edge is constrained from motion in the horizontal direction. In this study, the mesostructure of the concrete is assumed to comprise only of two phases viz., aggregate and mortar. A generic image of a specimen with aggregate volume ratio $\mu_{p}=60 \%$ with $2 \%$ porosity is shown in Fig. 9 . The aggregate phase is denoted in black whereas the mortar phase is denoted in grey.

The hybrid quadtree-polygonal mesh of a generic specimen is shown in Fig. 10a. The color of each phase is used as the homogeneity criterion during mesh generation. In order to preserve the full resolution of the image, the size of the smallest cell is set to 1 pixel when the quadtree decomposition was applied. The scaling between the image and the physical size of the specimen is $1: 1$, i.e. $(1$ pixel $=1 \mathrm{~mm})$. The size of the image is $128 \times 128$ pixels. Fig. 10b shows the quadtree mesh after fragmentation and insertion of interface elements. The interface elements are identified by color i.e. red along the aggregate-mortar interface; blue along the aggregate-aggregate interface; and yellow along the mortar-mortar interface. The inset in Fig. 9 shows a close up of interface elements in a the quadtree mesh.

The traction-separation laws adopted for the interface elements depend on the position of the interface elements viz. the aggregate-aggregate interface, the mortar-mortar interface or 


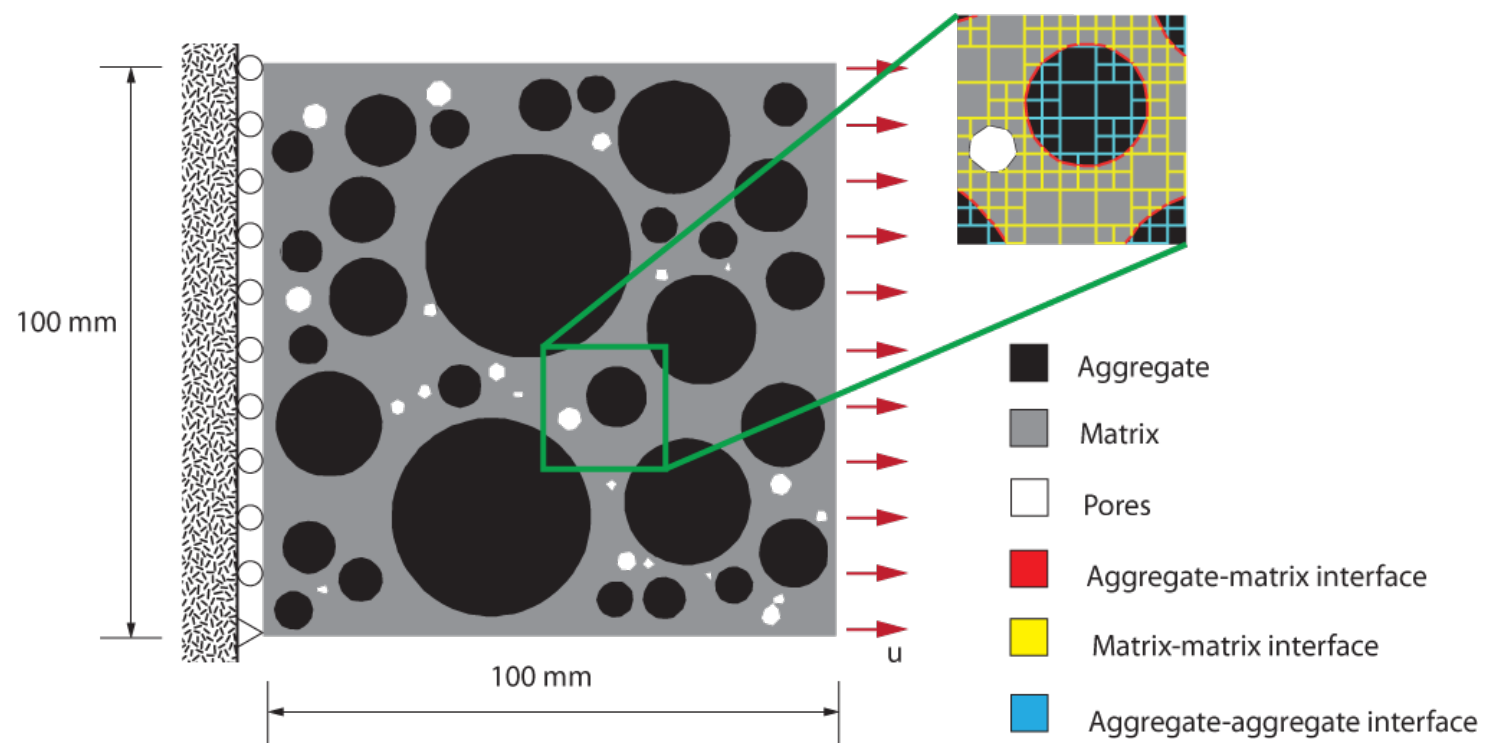

Figure 9: Concrete specimen subjected to uniaxial tension.

Table 1: Material properties adopted for different phases in the concrete mesostructure.

\begin{tabular}{ccccc}
\hline Component & $E(\mathrm{GPa})$ & $\nu$ & $f_{t}(\mathrm{MPa})$ & $G_{f}(\mathrm{~N} / \mathrm{mm})$ \\
\hline \hline Aggregate & 37 & 0.2 & - & - \\
Mortar & 20 & 0.2 & - & - \\
Aggregate-aggregate interface & 37 & - & 15 & 0.16 \\
Mortar-mortar interface & 20 & - & 3.8 & 0.09 \\
Aggregate-mortar interface & 18 & - & 1.9 & 0.045 \\
\hline
\end{tabular}


the aggregate-mortar interface. The material parameters used for the aggregate and mortar phases and to define the traction-separation laws are a source of ambiguity. The information that is reported in the literature is diverse. For example, different assumptions have been adopted for the behaviour of the aggregate phase. Trawiński et al. [47] and Rodrigues et al. [32] assumed that the aggregates do not fracture whereas Zhang et al. [57] and Benkemoun et al. [2] allowed fracture through aggregates. Different values have also been reported for the tensile strength $f_{t}$ for the mortar phase and the aggregate-mortar interface. In the former, the range of $f_{t}$ reported is between $2.6 \mathrm{MPa}$ and $6 \mathrm{MPa}$; whereas for the aggregate-mortar phase, $f_{t}$ between $0.5 \mathrm{MPa}$ and $3 \mathrm{MPa}$ has been reported $[36,48,2,32,47,37]$. The material properties adopted for the various phases of the concrete mesostructure in this study is shown in Table 1. The magnitudes of these parameters are selected to be within the range of the values previously reported in the literature. It is noted that the aggregate-mortar interface is the weakest component in the concrete mesostructure and is assumed to have half the tensile strength and fracture energy of the interface elements at the matrix-matrix interface e.g. $[32,17]$. It is also assumed that the cracks cannot propagate through the aggregates. This is achieved by assigning a reasonably high value of tensile strength and fracture energy to the aggregate-aggregate interface.

The traction-separation law in the normal direction is assumed to follow from the exponential relation reported by Karihaloo [18]

$$
\sigma=f_{t} e^{-4.6052 \frac{w}{w_{c}}}
$$

where the critical crack opening $w_{c}$ is estimated as

$$
w_{c}=\frac{4.6517 G_{f}}{f_{t}}
$$

The shear strength $f_{s}$ and shear fracture energy $G_{f s}$ required for the traction-separation law in the tangential direction are assumed to be $0.3 f_{t}$ and $0.3 G_{f}$, , respectively. The softening branch follows an exponential law as that in the normal direction.

The size of the quadtree mesh varies depending on the aggregate size distribution and the aggregate volume ratio. Generally, the number of cells in a mesh range between 2500 and 3800 and the number of nodes between 10000 and 16000. During the fracture simulations, however, the initial SBFEM stiffness matrices are pre-calculated and stored for all the 32 possible master quadtree cells (16 possible master cells in each phase, aggregate and mortar). These constitute the bulk of cells in the mesh. Additional computations of stiffness matrices are required only for the irregular cells located on the boundary or between dissimilar materials. 


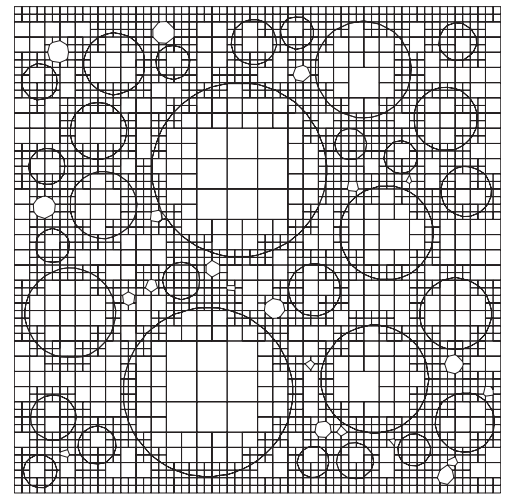

(a) hybrid quadtree mesh

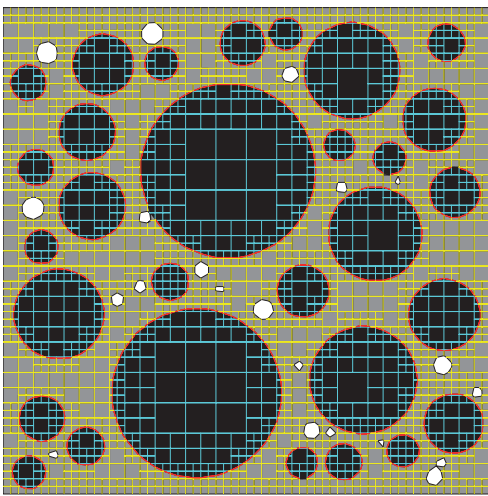

(b) hybrid quadtree mesh showing interface elements

Figure 10: Generic quadtree mesh generated from a specimen with $\mu_{p}=60 \%$.

The nonlinear analysis is carried out by adopting a standard displacement control scheme. During pre-peak response, discrete cohesive constitutive model relating stresses and relative displacement requires the use of very high elastic stiffness to preclude relative displacements in the interfaces before failure. This can be a source of numerical instabilities depending on the assumed value for the elastic stiffness. The elastic stiffness is identified by adopting a critical value of $1 \times 10^{-4} \mathrm{~mm}$ so that the initial stiffnesses of the aggregate-aggregate, mortarmortar and aggregate-mortar are $150000 \mathrm{MPa} / \mathrm{mm}, 38000 \mathrm{MPa} / \mathrm{mm}$ and $19000 \mathrm{MPa} / \mathrm{mm}$, respectively. To avoid numerical instabilities in the solution of the nonlinear system of equations due to the formation of multiple cracks in heterogeneous materials (usually during the late post peak regions), the change of the norm of displacement increments between each iteration to predict if the equilibrium iterations will converge. If the change in the norm of displacement between ten successive iterations is too small, the solution is terminated and the results from the final load converged load step is recovered.

\subsubsection{Influence of aggregate volume Ratio}

The influence of the aggregate volume ratio on the fracture parameters of in the concrete mesostructure under uniaxial tension is studied. Square concrete specimens with aggregate volume ratio $\mu_{p}$ of $40 \%, 50 \%$ and $60 \%$ are considered. The aggregate size distribution is chosen based on the nominal sieve sizes specified in Australian Standard AS 2758.0 Cl 3.5 [1] and is $[38.3 \mathrm{~mm}, 26.5 \mathrm{~mm}, 19 \mathrm{~mm}, 13.2 \mathrm{~mm}, 9.5 \mathrm{~mm}, 6.7 \mathrm{~mm}]$. For each $\mu_{p}$ considered, five samples are generated.

The individual stress-displacement responses of the concrete specimens are shown in Fig. 11. The pre-peak responses of all the specimens are similar and are consistent with the 


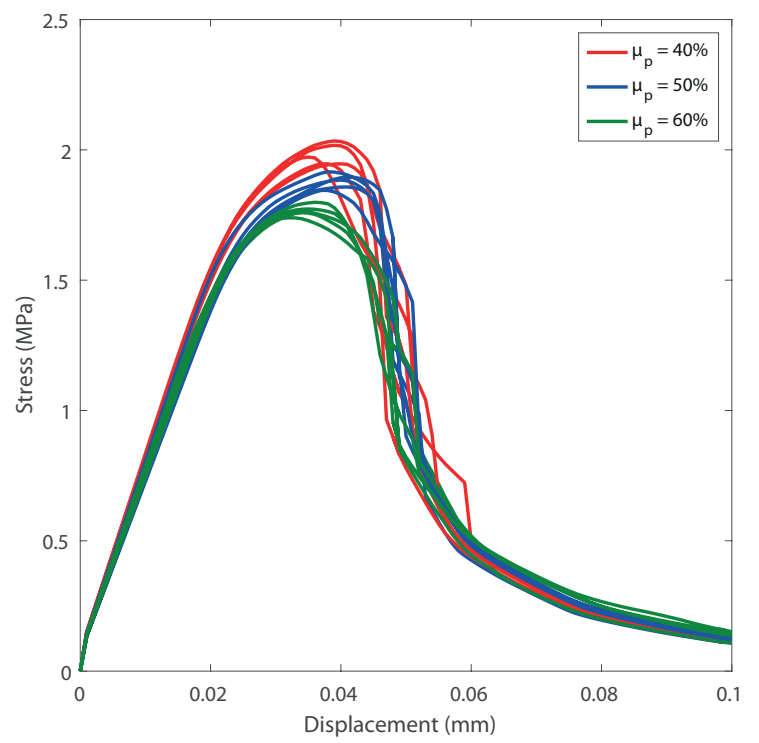

Figure 11: Stress-displacement response of concrete specimens with different aggregate volume ratio.

simulations reported by Rodrigues et al. [32]. The stress here is defined as the ratio of the sum of the reaction forces at the left edge of the specimen to the specimen's cross sectional area.

The predicted peak stress was between 1.74 MPa and 2.0 MPa depending on the aggregate volume ratio. The predicted values are in proportion to typical concrete tensile strengths reported in the literature e.g. [28] depending on the water-to-cement ratio of the mix. There is a clear dependence on the predicted load carrying capacity with the tensile strength adopted for the interface transition zone. The load carrying capacity was close to the tensile strength at the aggregate-mortar interface, which was $1.9 \mathrm{MPa}$. Variations between specimens with the same $\mu_{p}$ is observed. The predicted peak stress was $1.95 \mathrm{MPa}$ to $2.03 \mathrm{MPa}$ in specimens with $\mu_{p}=40 \%, 1.85 \mathrm{MPa}$ to $1.91 \mathrm{MPa}$ in specimens with $\mu_{p}=50 \%$ and $1.74 \mathrm{MPa}$ to $1.79 \mathrm{MPa}$ in specimens with $\mu_{p}=60 \%$. However, the observation that concrete specimens with lower $\mu_{p}$ lead to a higher load carrying capacity is consistent between specimens with different $\mu_{p}$. This result agrees with the numerical results in previous studies e.g. [48, 31, 32] which assumes that the aggregate-mortar interface is weakest in the concrete mesostructure.

Specimens with higher $\mu_{p}$ are observed to result in a higher number of interface elements along the aggregate-mortar interface. As the aggregate-mortar interface is weakest in the concrete mesostructure, a higher aggregate volume fracture promotes the coupling along these interfaces, which is conducive to the formation of cracks and therefore, contributes to the reduced load carrying capacity of these specimens [20]. This effect can be explained by examining the relative displacements of the interface elements at peak load of generic 


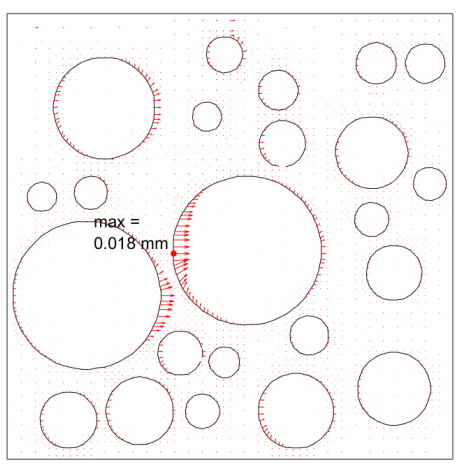

(a) $\mu_{p}=40 \%$

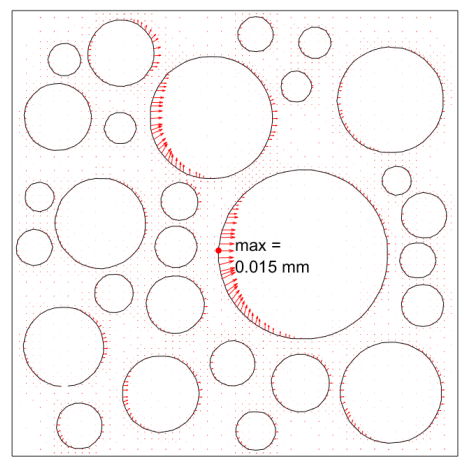

(b) $\mu_{p}=50 \%$

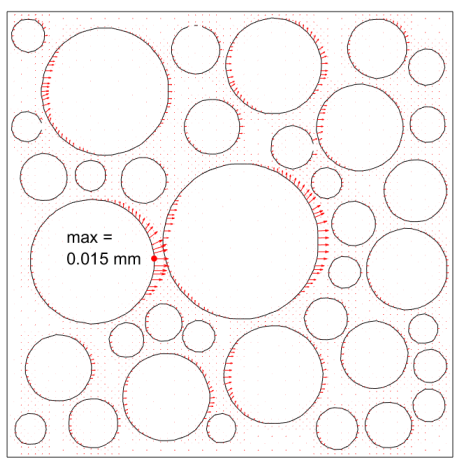

(c) $\mu_{p}=60 \%$

Figure 12: Development of micro fractures (in red) at peak load.

specimens with different $\mu_{p}$ as shown in the plots in Fig. 12. Although the magnitudes of maximum relative displacements is comparable between specimens with different $\mu_{p}$, specimens with a larger aggregate volume ratio is observed to have more regions in which the crack bridging mechanisms are active (indicated by the presence of more identifiable magnitudes of relative displacements around the aggregates as shown in Fig. 12c). This led to the lower load carrying capacity of these specimens.

It is worth noticing here that the mode mixity $\alpha$ defined as

$$
\alpha=\frac{f_{s}}{f_{t}}=\frac{G_{f s}}{G_{f}}
$$

has some degree of influence over the predicted peak stress of the specimens. This can be demonstrated by investigating a $\mu_{p}=60 \%$. concrete specimen with the same aggregate size distribution considered in the aforementioned parametric study. The specimen is subjected to uniaxial tension and the stress-displacement responses are recorded for three different mode mixity $\alpha=0.30, \alpha=0.45$ and $\alpha=0.60$. The plots of specimen stress versus applied displacement in Fig. 13 show that the specimen's peak stress increases with increasing $\alpha$. This observation can be attributed to the orientation of the interface elements in the mesh. The inclined interface elements undergo mixed-mode deformation. Depending on the inclination angle of each element, the mode mixity can have an effect on the overall deformation of the specimen. An assumption of a high mode mixity increases the cohesive forces in these interface elements resulting in a specimen that can sustain a higher load.

It is also observed that there is more variance in the post-peak response for specimens with a smaller $\mu_{p}$ as compared with specimens with larger $\mu_{p}$. This can be attributed to larger the degree of heterogeneity of the mesostructure in the specimens with lower aggregate 


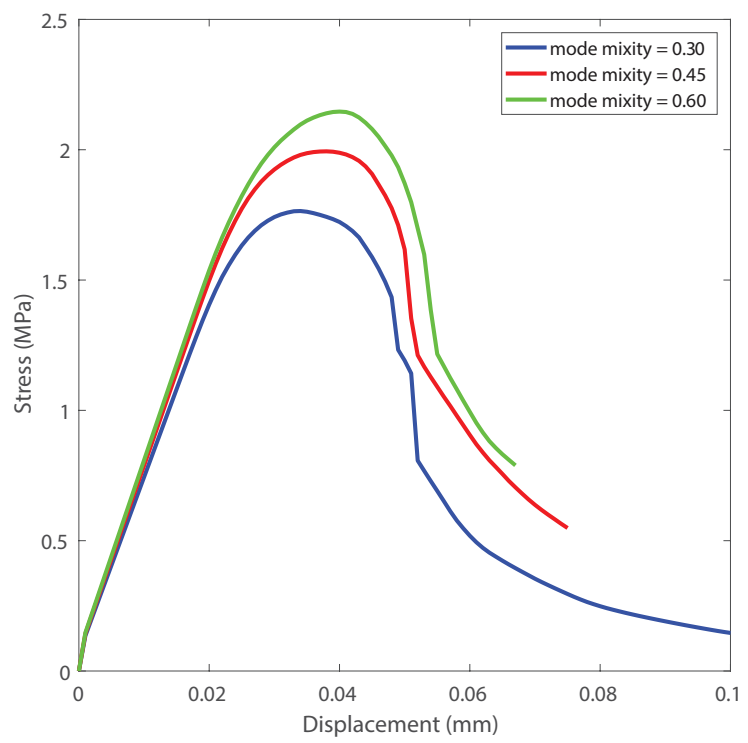

Figure 13: Effect of mode mixity $\alpha$ on the predicted peak stress of a generic concrete specimen.

volume ratio. In specimens with a higher $\mu_{p}$, the aggregates fill up most of the space inside the mesostructure, which results in a more homogeneous structure compared with specimens with a lower $\mu_{p}$. The increased randomness of aggregate placement in specimens with low aggregate volume ratio ultimately induces a higher variance in the predicted load deflection responses.

Fig. 14 shows the final predicted crack paths for generic specimens having different aggregate-to-matrix ratio $\mu_{p}$. For all the 15 specimens considered in this parametric study, the failure of the specimens is characterised by the development of a major crack that cuts through the specimen. The position of the macrocrack is random and depends on the arrangement of the aggregates in the concrete mesostructure. In all the specimens, the development of the fracture surfaces is evident only when the load-displacement response enters the region near peak load. During peak load, the initiation of small cracks can be observed along the interfaces of the aggregates. These are particularly evident along the larger sized aggregates as can be observed in the plot of the relative displacements of the interface elements in Fig. 12.

Compared with the smaller sized aggregates, the interface transition zones surrounding the large-sized aggregates are longer. Collectively, these regions are therefore, weaker compared with those surrounding the smaller aggregates. Cracks are therefore, more likely to initiate near the larger aggregates. As the softening phase progresses further, these cracks grow in length and coalesce with the other cracks in their vicinity. This subsequently led to the development of a major crack. At this stage, the cracks along the aggregates that 


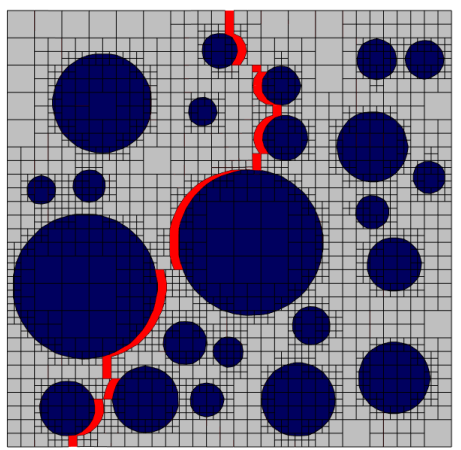

(a) $\mu_{p}=40 \%$

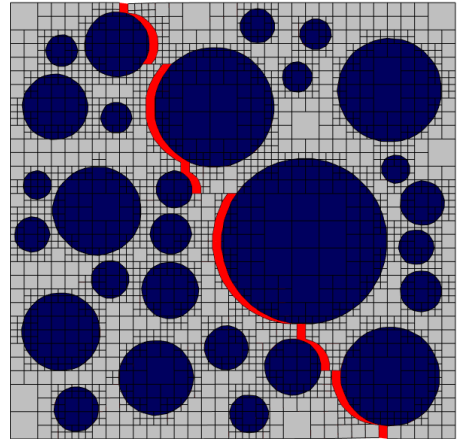

(b) $\mu_{p}=50 \%$

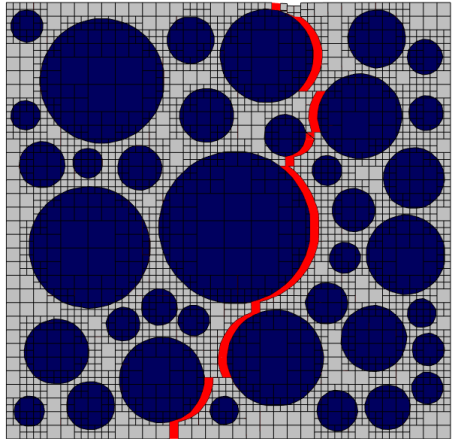

(c) $\mu_{p}=60 \%$

Figure 14: Final predicted crack paths of generic specimens for different $\mu_{p}$ scaled 20 times.

are positioned far away from the major crack begin to close as the interface transition zones in these regions experience unloading. The specimens ultimately fail when the major crack propagates through the entire length of the specimen.

Irrespective of a specimen's aggregate volume ratio, the influence of the heterogeneity of the aggregate arrangement on the final crack path, is evident. Fig. 15 shows the predicted crack paths for the remaining 4 specimens with $\mu_{p}=50 \%$. Although the paths of the failure macrocrack crack differ from specimen to specimen, they were observed to concentrate along the interface elements surrounding the large sized aggregates, highlighting the influence of the heterogeneity of the mesostructure in the predicted fracture paths.

\subsubsection{Influence of Aggregate Size Distribution}

The influence of the aggregate size distribution on the mesoscale fracture characteristics of concrete under direct tension (Fig. 9) is studied next. Square concrete specimens with aggregate volume fraction $\mu_{p}=50 \%$ are generated considering three different aggregate size distributions as follows:

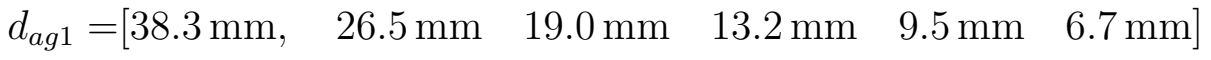

$$
\begin{aligned}
& d_{a g 2}=\left[\begin{array}{lllll}
26.5 \mathrm{~mm} & 19.0 \mathrm{~mm} & 13.2 \mathrm{~mm} & 9.5 \mathrm{~mm} & 6.7 \mathrm{~mm}
\end{array}\right] \\
& d_{a g 3}=\left[\begin{array}{llll}
19.0 \mathrm{~mm} & 13.2 \mathrm{~mm} & 9.5 \mathrm{~mm} & 6.7 \mathrm{~mm}
\end{array}\right]
\end{aligned}
$$

Each aggregate distribution is distinguished by the maximum aggregate diameter $d_{\max }$ in the concrete mix. For each aggregate size distribution considered, 5 samples are generated.

Fig. 16 shows the predicted structural response obtained from the samples having vary- 


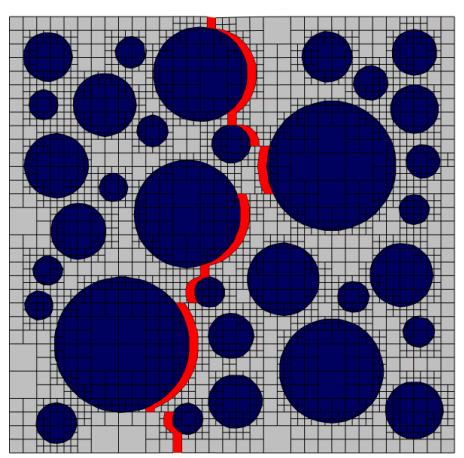

(a) Specimen 2

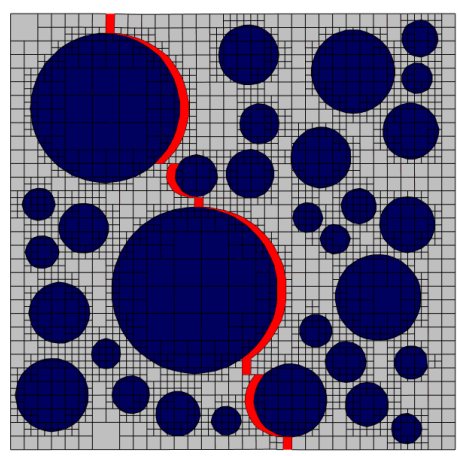

(c) Specimen 4

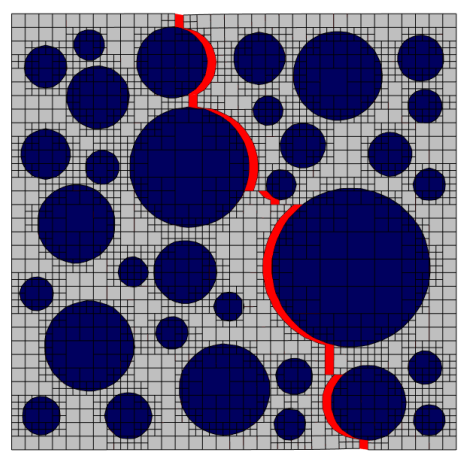

(b) Specimen 3

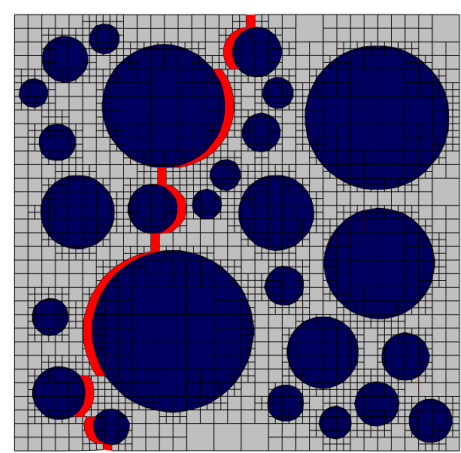

(d) Specimen 5

Figure 15: Final predicted crack pattern for four different specimens with $\mu_{p}=50 \%$ scaled 20 times.

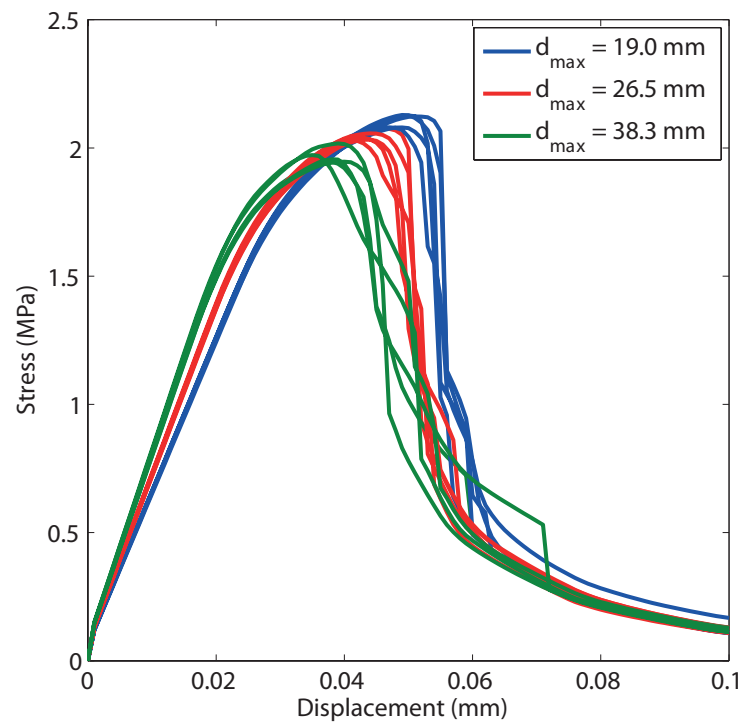

Figure 16: Stress-displacement response of concrete specimens with different aggregate distribution. 
ing aggregate distribution. It is observed that the load carrying capacity decreases with increasing aggregate size. The predicted peak stress was $2.08-2.13 \mathrm{MPa}$ for specimens with $d_{\max }=19.0 \mathrm{~mm}, 2.03-2.07 \mathrm{MPa}$ for specimens with $d_{\max }=26.5 \mathrm{~mm}$ and $1.95-2.02 \mathrm{MPa}$ for specimens with $d_{\max }=38.3 \mathrm{~mm}$. Also observed in Fig. 16 is the less pronounced softening branch of the specimens with smaller $d_{\max }$. This is clearly observed in the load-displacement responses of specimens with $d_{\max }=19.0 \mathrm{~mm}$ and $d_{\max }=26.5 \mathrm{~mm}$ by a sharp decline in the load carrying capacity immediately after the peak load has been reached. This trend is less obvious in specimens with $d_{\max }=38.3 \mathrm{~mm}$ where the softening phase is more pronounced. Numerical simulations reported by Rodrigues et al. [32], Du et al. [9] and Huang and Li [15]; and experimental observations reported by Hordijk [14] support the current findings.

Rodrigues et al. [32] and López et al. [23] attributed the less pronounced softening branch to the increased crack bridging mechanisms from the crack interface interactions around the larger sized aggregates. This increases the residual strength resulting in more energy dissipated from the fracture evolution during the softening phase and thereby, an increased area under the curve in this region. The decrease in the observed peak stress in specimens with larger aggregate sizes is attributed to the concentrated localised failure around the few large sized aggregates. Compared with samples with smaller aggregates, the relative displacement that develops around the large aggregates is larger despite reaching the peak load at a lower applied displacement $u$ (see Fig. 16); thereby inferring a lower cohesive force in these regions. This can be identified by comparing maximum crack opening displacements of the interface elements in the mesh at peak load in the quiver plots shown in Fig. 17 or by comparing the magnitude of displacement discontinuity between the aggregate and matrix phases in the displacement contour plots shown in Fig. 17. In specimens with $d_{\max }=19.0 \mathrm{~mm}$, the average maximum crack opening displacement was $0.009 \mathrm{~mm}$, whereas it was $0.011 \mathrm{~mm}$ and $0.018 \mathrm{~mm}$ in the specimens with $d_{\max }=26.5 \mathrm{~mm}$ and $d_{\max }=38.3 \mathrm{~mm}$, respectively.

Fig. 18 shows the final crack patterns obtained from typical specimens with $d_{\max }=$ $38.2 \mathrm{~mm}, d_{\max }=26.5 \mathrm{~mm}$ and $d_{\max }=19.0 \mathrm{~mm}$. The failure macrocrack is observed to develop along the aggregate-mortar interface. In the specimens with larger aggregate diameter, the failure crack tends to develop along the larger sized aggregates. This is supported by the observation of the increased crack bridging mechanisms at the interface transition zones along the boundaries these aggregates (Fig. 17). In specimens with a smaller aggregate diameter, the distribution of the aggregates resulted in a more homogeneous structure. Although the failure macrocrack still propagate through the weaker interface transition zones between the mortar and aggregate phase, the congregation of the crack along the larger sized aggregates is not obvious as compared with the specimens with larger aggregate diameters. In these specimens, the failure crack tends to develop in a region of closely packed aggregates where 


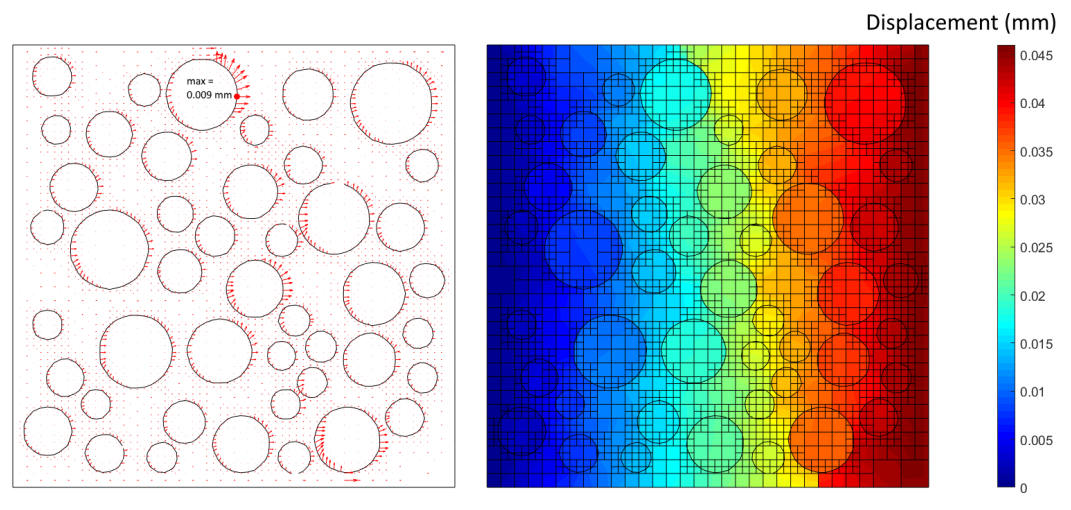

(a) $d_{\max }=19.0 \mathrm{~mm}$
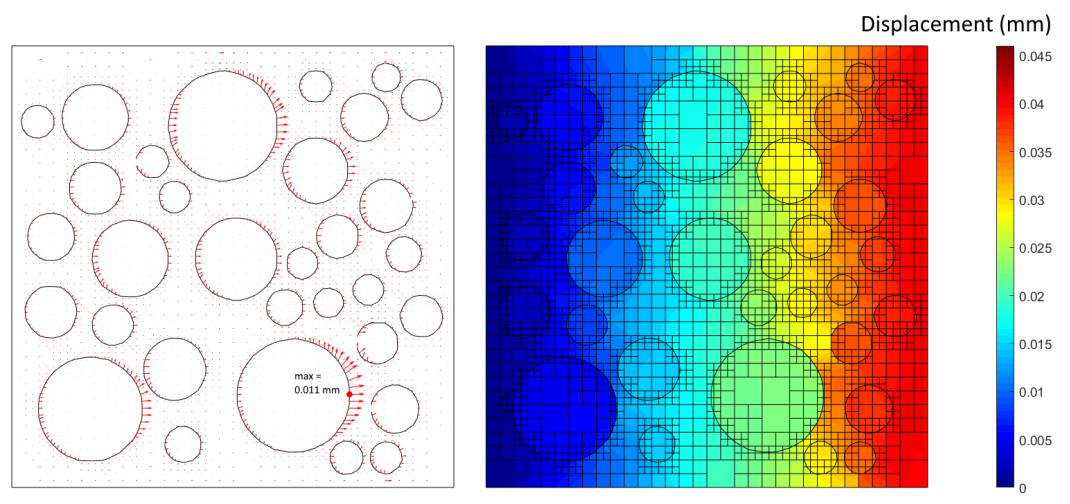

(b) $d_{\max }=26.5 \mathrm{~mm}$
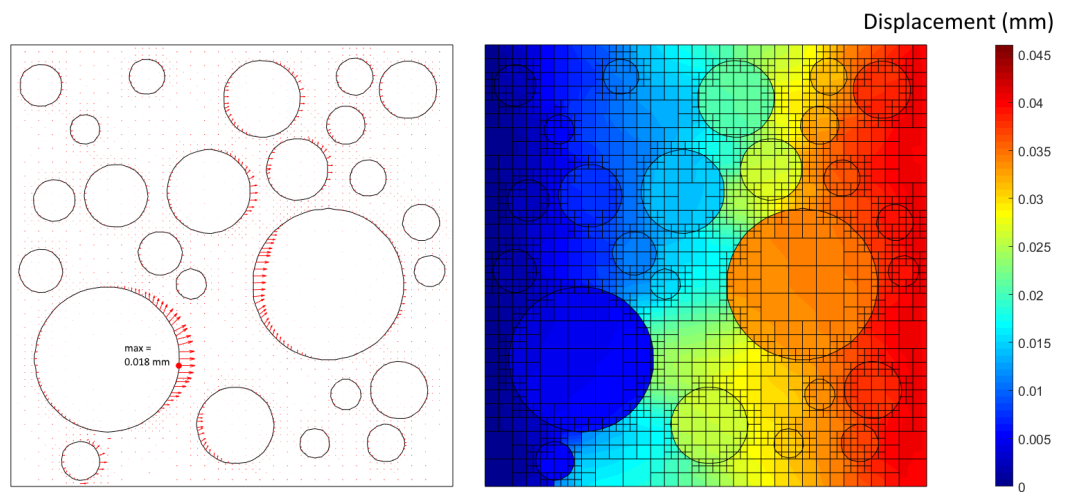

(c) $d_{\max }=38.3 \mathrm{~mm}$

Figure 17: Displacement contour of typical specimens with different $d_{\max }$ at peak load. 
the cumulative effect of the crack bridging mechanisms lead to the coalescence of the small cracks in the vicinity of the small sized aggregates e.g. Fig. 18c.

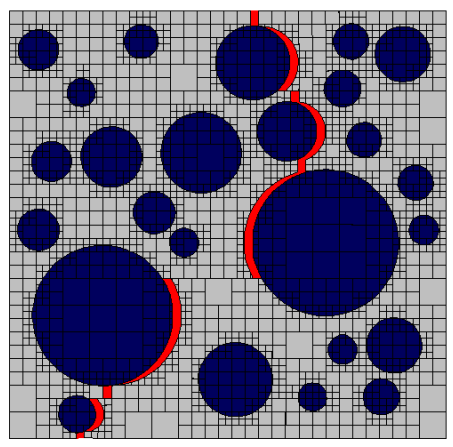

(a) $d_{\max }=38.3 \mathrm{~mm}$

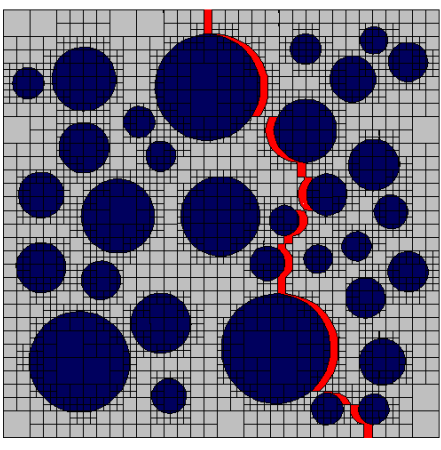

(b) $d_{\max }=26.5 \mathrm{~mm}$

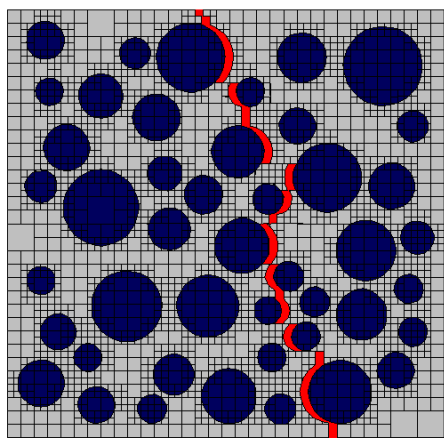

(c) $d_{\max }=19.0 \mathrm{~mm}$

Figure 18: Final predicted crack pattern for typical specimens with different magnitudes of $d_{\max }$ magnified 20 times.

\subsubsection{Influence of Specimen Porosity}

The effect of porosity on the fracture characteristics of concrete mesostructure is investigated next. Concrete specimens with aggregate volume ratio $\mu_{p}=50 \%$ are generated considering different levels of porosity. Porosity can be introduced during meso-scale image generation in a similar manner in which the aggregates are generated. The air gaps are assumed to be circular in shape with diameter randomly sampled between $3.0 \mathrm{~mm} \leq d_{\text {pore }} \leq 5.0 \mathrm{~mm}$. The operation to include the air gaps in the specimen requires only filling up an admissible location with a different color (in this case, white) and is initiated after aggregate generation. Three different porosity levels are considered viz., 1\%, $2 \%$ and $3 \%$. For each porosity level considered, 5 samples are generated. The load carrying capacity of the porous specimens are compared with specimens with zero porosity.

Fig. 19 shows the influence of porosity on the load carrying capacity of the specimens. A decrease in the tensile load carrying capacity is observed with increasing porosity. The range of predicted peak stress is $1.86-1.92 \mathrm{MPa}$ for specimens with zero porosity, $1.81-1.88$ MPa for specimens with $1 \%$ porosity, $1.69-1.74 \mathrm{MPa}$ for specimens with $2 \%$ porosity and $1.55-1.63 \mathrm{MPa}$ for specimens with $3 \%$ porosity. The loss in load carrying capacity is quite significant considering an average loss of $10 \%$ when there is an increase of only $2 \%$ in the porosity.

The decrease in tensile load carrying capacity with increasing porosity can be attributed to the decreased surface area available to sustain the applied load. This creates an environ- 


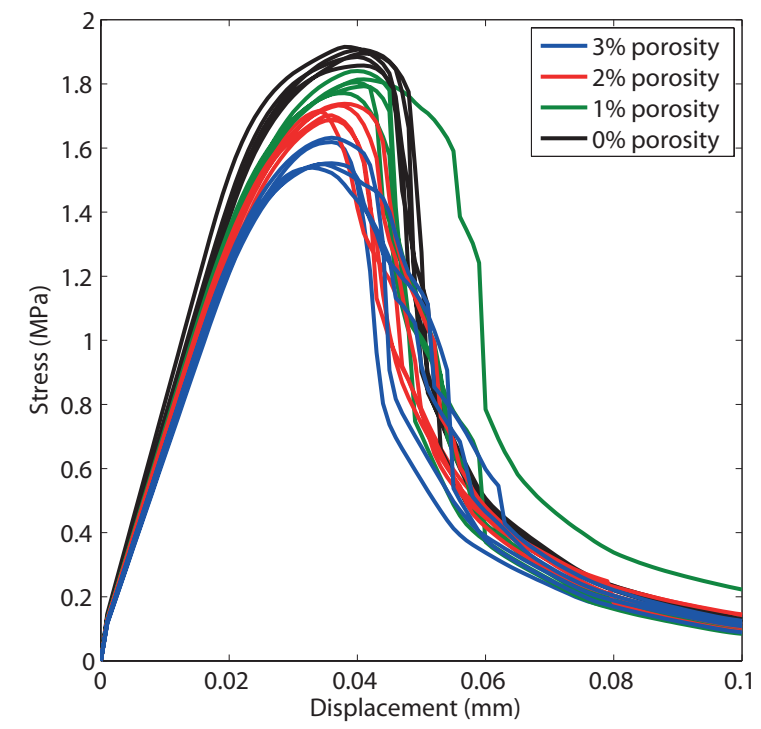

Figure 19: Stress-displacement response of concrete specimens with different porosity levels.

ment which is conducive to the evolution of fractures. The regions in which voids are present offer no resistance to the propagation of cracks and promote the coalescence of the small cracks that initiate at the interface transition zone of the surrounding aggregates. Fig. 20 shows the final crack patterns of typical concrete specimens with different porosity levels. It can be identified from these figures that the failure macrocrack of the specimen propagates through the voids wherever possible, following the path of least resistance to fracture the specimen. A distinction can be observed when comparing the fracture paths with a specimen with zero porosity (e.g. Fig. 14b). For the non-porous specimens, the fracture path is generally identified to follow the boundary of the aggregates. In the porous specimens, the presence of the voids facilitates fracture through the matrix in the vicinity of the voids.

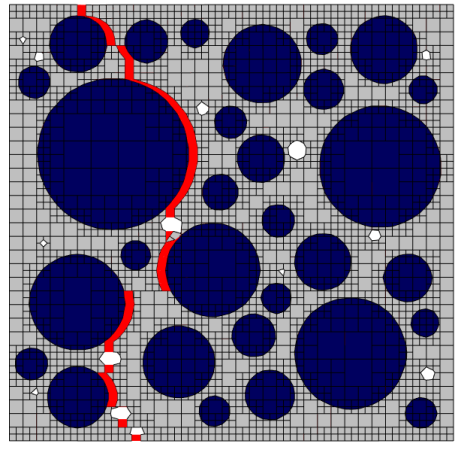

(a) $1 \%$ porosity

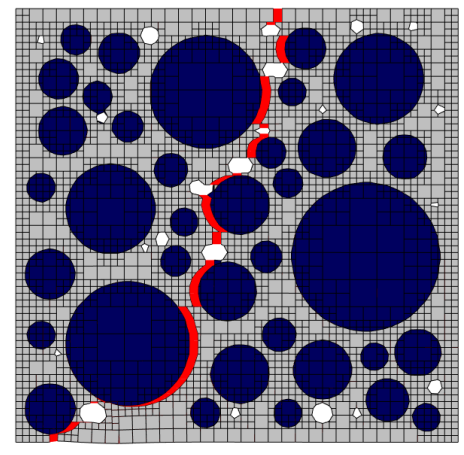

(b) $2 \%$ porosity

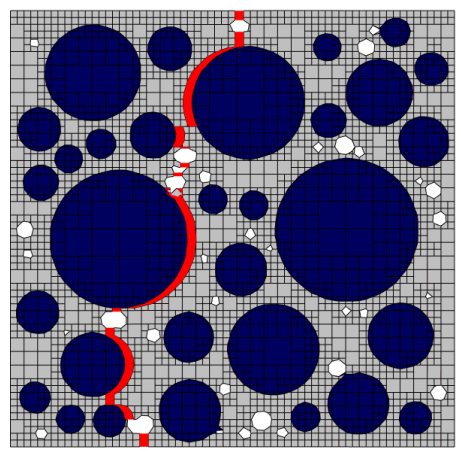

(c) $3 \%$ porosity

Figure 20: Final predicted crack pattern for typical different specimens with porosity magnified 20 times. 


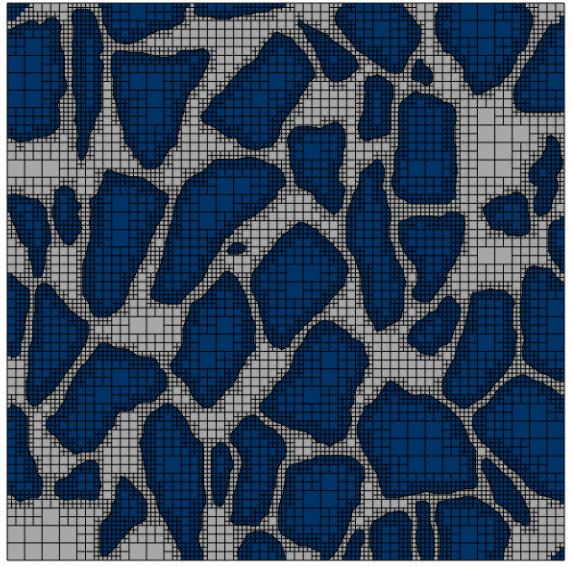

(a) specimen 1

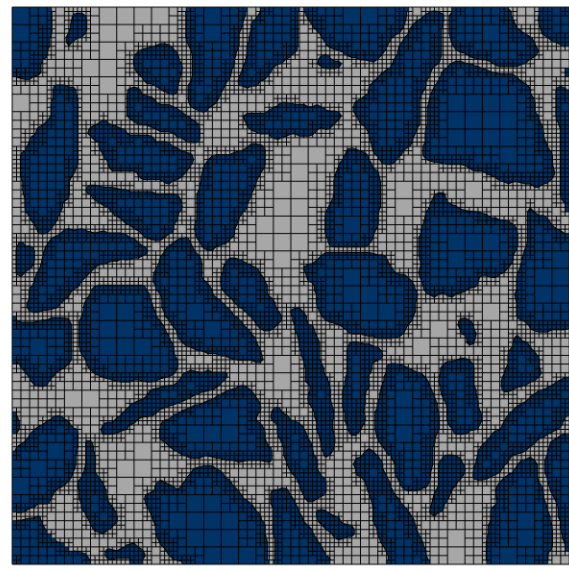

(c) specimen 3

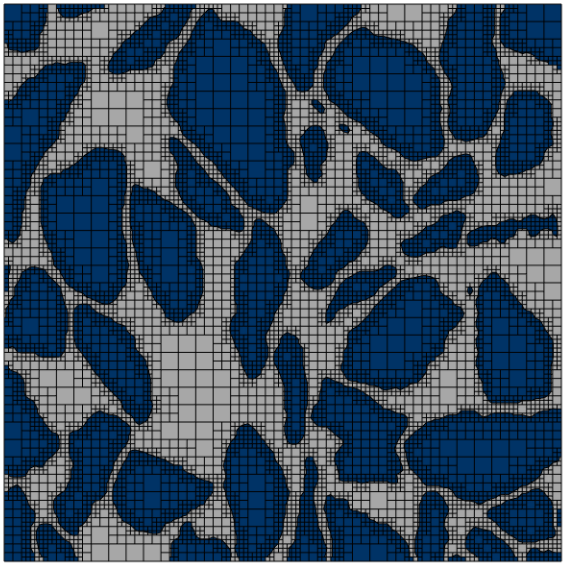

(b) specimen 2

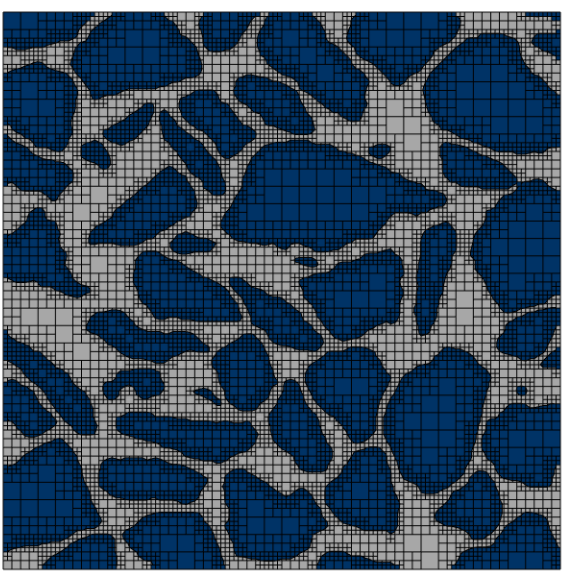

(d) specimen 4

Figure 21: Concrete specimens obtained from XCT scanning subjected to uniaxial tension.

\subsection{Mesoscale Fracture Modelling of Concrete Specimens from XCT Scans}

To demonstrate the application of the developed approach on the modelling of realistic concrete mesostructure, the XCT images of concrete specimens obtained from the study of Ren et al. [31] are considered. The hybrid quadtree polygon meshes of each specimen's meso-structure are shown in Fig. 21. The meshes are generated from images having a size of $256 \times 256$ pixels. The smallest and largest pixel size adopted for quadtree discretisation are 2 and 32, respectively. The smoothing operation described in Section 2.2 is then applied to remove the jagged edges resulting from a purely quadtree decomposition along the edges between the aggregates and the mortar. The aggregate volume ratio of Specimens 1, 2, 3 and 4 are $61 \%, 57 \%, 56 \%$ and $57 \%$, respectively. 


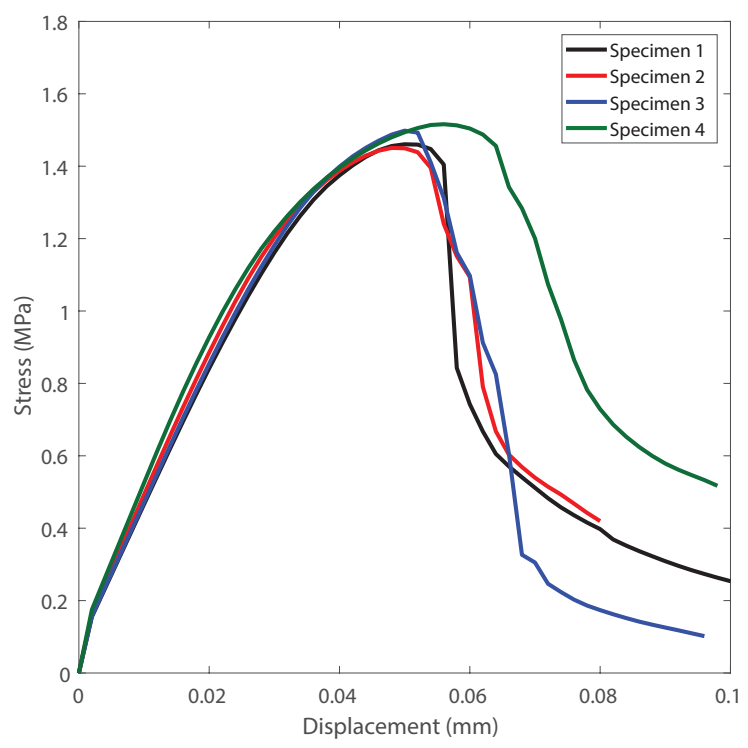

Figure 22: Stress-displacement response of concrete specimens obtained from XCT scans.

All the specimens are constrained and subjected to direct tension similar to the conditions shown in Fig. 9. The material properties shown in Table 1 are adopted for the different phases in the concrete mesostructure. The softening laws in both normal and tangential directions follow from that described in Section 5.1.

Fig. 22 shows the stress-displacement responses of the four concrete specimens. The predicted peak stress of the specimens are between $1.46-1.52 \mathrm{MPa}$. Fig. 23 shows the predicted failure crack in each of the four specimens considered. It is evident from Fig. 22 and Fig. 23 that the heterogeneity of the concrete meso-structure has a huge effect on the predicted fracture patterns. The failure patterns of the four specimens are reminiscence of the failure patterns reported by Wang et al. [48] and Ren et al. [31]. The specimens either fail due to the development of a single macrocrack viz.. specimens 1 and 3 or fail due to the development of two macrocracks viz. specimens 2 and 4 . The more rapid drop in the strength of specimens that fail due to the development of a single macrocrack is evident in the stressdisplacement responses of specimens 1 and 3 in Fig. 22, when compared with specimens that fail due to the development of two macrocracks viz. specimens 2 and 4 . This observation is consistent with the results of Wang et al. [48] and Ren et al. [31], and can be attributed to the lack of crack bridging mechanisms in specimens where two macrocrack develops, leading to a high residual load carrying capacity. The residual load carrying capacity can be observed to also depend on the length of the developed macrocrack. The longer the macrocrack, the higher the residual load carrying capacity. This is especially obvious in specimen 4, where the failure macrocrack is observed to be longer than the other specimens. Consequently, this would mean that the development of the macrocrack requires the expenditure of more 


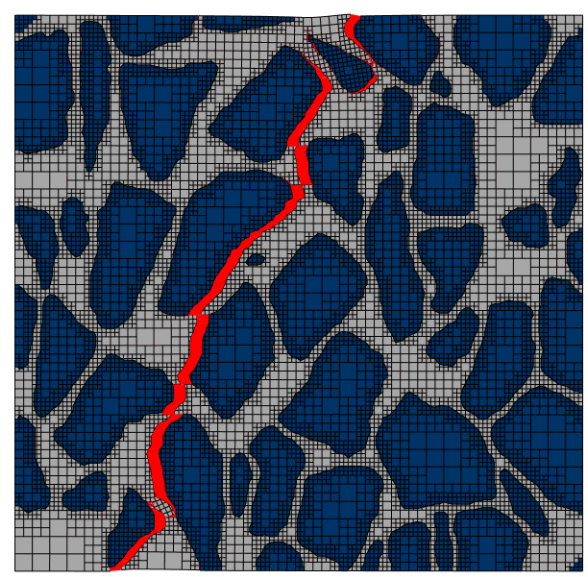

(a) specimen 1

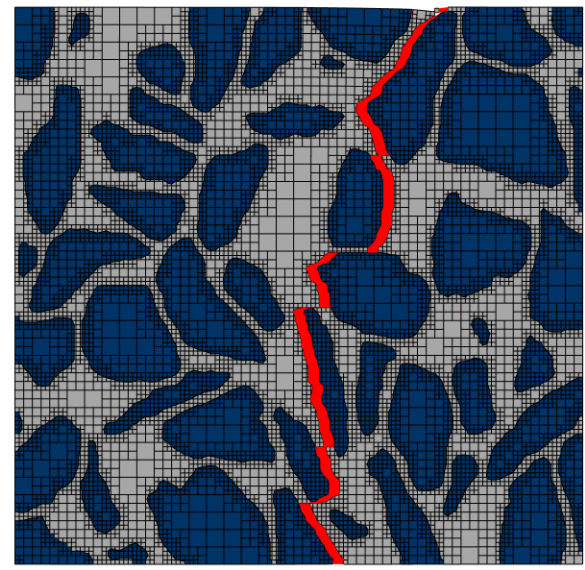

(c) specimen 3

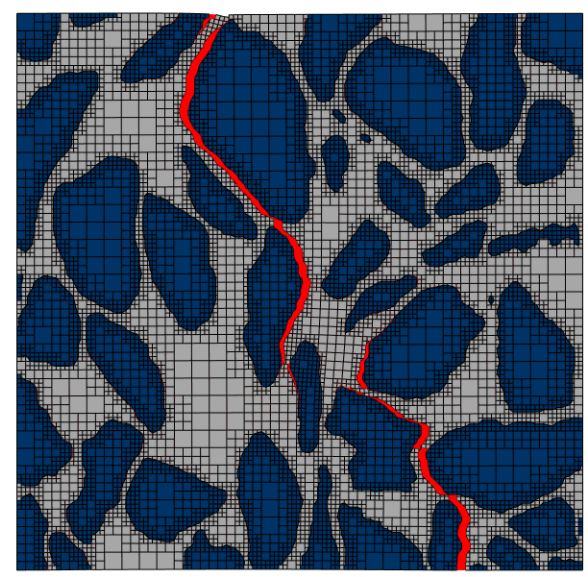

(b) specimen 2

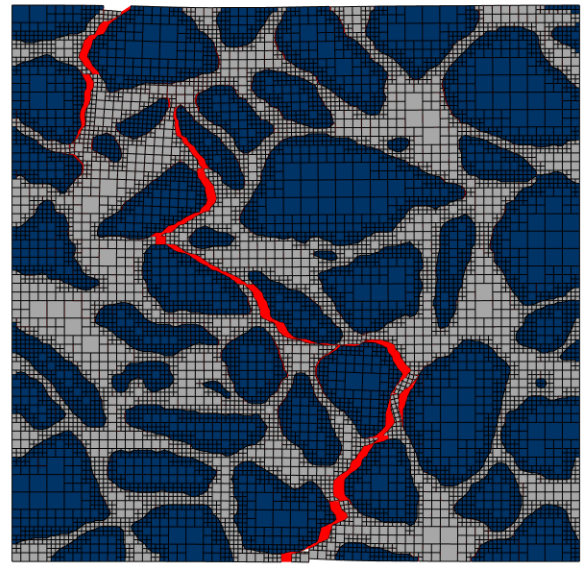

(d) specimen 4

Figure 23: Final predicted crack patterns for XCT imaged specimens magnified 20 times. 
energy and hence a higher residual load carrying capacity. The results of this analysis clearly indicates the strong influence of the heterogeneity of the mesostructure on the peak stress and fracture pattern of concrete.

\section{Conclusions}

Meso-scale fracture in concrete was modelled using an automatic image-based, hybrid quadtreepolygon SBFEM framework. The flexibility of the SBFEM to directly model arbitrary sided polygons enables the geometric features in the concrete meso-structure to be adequately represented without the need for very fine discretisations. This was achieved by adopting a combination of quadtree cells and arbitrary sided polygons.

Automatic mesh generation from digital images was achieved by adopting a balanced quadtree decomposition. A smoothing procedure which splits the quadtree cells into two arbitrary sided polygons is applied to improve the boundary representation of the aggregates and air voids in the mesostructure. This operation eliminates the jagged edges around the aggregates and the boundaries of pores resulting from a purely quadtree decomposition. It also removes the requirement for an unnecessary fine mesh in these regions, that would otherwise be required for a more accurate discretisation of irregular boundaries if a pure quadtree mesh was adopted. The SBFEM models each quadtree cell directly as an arbitrary sided polygon irrespective of the presence of hanging nodes between adjacent quadtree cells of different sizes. The polygonal cells along the aggregate or air void boundaries, which are products of the smoothing operation are directly modelled by the SBFEM.

The fracture process zone within the meso-structure is modelled using pre-inserted zerothickness interface elements in the mesh following a mesh fragmentation process. Appropriate constitutive laws are assigned to the interface elements depending on their positions in the mesh e.g. aggregate-to-aggregate, matrix-to-aggregate.

Parametric studies were carried out on graded distributions of representative graded concrete meso-structures subjected to direct uniaxial tension. These showed that:

1. The developed technique was capable of reproducing the typical fracture characteristics of concrete observed in numerical and experimental studies reported in the literature $[14,23,32]$ e.g. the reduction of load carrying capacity with increased aggregate diameter, aggregate volume ratio and porosity; the more pronounced softening branch in specimens with larger aggregate diameter.

2. The ultimate tensile load carrying capacity depends on the magnitude of the tensile strength of the interface elements used to model the interface transition zone between 
the aggregate and mortar phases.

3. The heterogeneity of the concrete mesostructure has a profound influence on the predicted load carrying capacity and the final predicted crack paths. The failure macrocrack has a tendency to develop and coalesce between aggregates with larger diameters or a congregate of closely spaced aggregates.

4. The reduction the load carrying capacity under direct uniaxial tension is attributed to the increased crack bridging mechanisms in specimens with larger aggregate diameters and also in specimens with larger aggregate volume ratio as the loading response progresses into the softening phase (Fig. 12 and Fig. 17).

Mesoscale fracture analyses of real concrete specimens obtained from XCT scans were carried out. The results showed mesoscale fracture characteristics of concrete under direct tension that are consistent with the results reported in the literature.

The proposed method adopt fixed meshes, which limits the number of available paths for fracture and possibly result in some biasing of the propagation directions. Even if triangular elements are used as in the context of the FEM, mesh biasing cannot be completely eliminated e.g. [5]. The bias in the propagation directions may be overcome by adopting an adaptive meshing techniques. This may be possible by combining the present approach with the general coordinate formulation with side face tractions to resolve the crack tip fields and determine the propagation directions from the stress field. The quadtree cells can be further remeshed into several polygons during crack propagation to avoid bias in the propagation direction.

It is also to be noted that although capable of producing pertinent qualitative results in the uniaxial tension test, the developed method is bound by the limitations of a twodimensional analysis. Representation of concrete mesostructure by planar segments is not representative of the actual three-dimensional morphologies of the concrete aggregates. As a result, a two-dimensional analysis is in capable of modelling the fracture pattern observed experimentally in cubic concrete specimens.

\section{References}

[1] AS2758, 2009. Aggregates and rock for engineering purposes.

[2] Benkemoun, N., Poullain, P., Khazraji, H. A., Choinska, M., Khelidj, A., 2016. Mesoscale investigation of failure in the tensile splitting test: size effect and fracture energy analysis. Engineering Fracture Mechanics 168, 242-259. 
[3] Bolander, J. E., Saito, S., 1998. Fracture analyses using spring networks with random geometry. Engineering Fracture Mechanics 61, 569-591.

[4] Bruggi, M., Casciati, S., Faravelli, L., 2008. Cohesive crack propagation in a random elastic medium. Probabilistic Engineering Mechanics 23, 23-35.

[5] Camacho, G. T., Ortiz, M., 1996. Computational modelling of impact damage in brittle materials. International Journal of Solids and Structures 33, 2899-2938.

[6] Cundall, P., Strack, O., 1979. A discrete numerical model for granular assemblies. Géotechnique 29, 47-65.

[7] D’Addetta, G. A., Kun, F., Ramm, E., 2002. Intheapplaspects of a discrete model to the fracture process of cohesive granular materials. Granular Matter 4, 77-90.

[8] Deeks, A. J., Wolf, J. P., 2002. A virtual work derivation of the scaled boundary finite element method for elastostatics. Computational Mechanics 28, 489-504.

[9] Du, X., Jin, L., Ma, G., 2014. Numerical modeling tensile failure behavior of concrete at mesoscale using extended finite element method. International Journal of Damage Mechanics 23, 872-898.

[10] Eláš, J., Stang, H., 2012. Lattice model modeling of aggregate interlocking in concrete. International Journal of Fracture 175, 1-11.

[11] Feng, Y. T., Owen, D. R. J., 2004. A 2d polygon/polygon contact model: algorithmic aspects. Engineering Computations 21, 265-277.

[12] Gerstle, W. H., Xie, M., 1992. Fem modelling of fictitious crack propagation in concrete. Journal of Engineering Mechanics ASCE 118, 416-434.

[13] Grassl, P., Jirásek, M., 2010. Meso-scale approach to modelling the fracture process zone of concrete subjected to uniaxial tension. International Journal of Solids and Structures 47, 957-968.

[14] Hordijk, D., 1992. Tensile and tensile fatigue behaviour of concrete; experiments, modeling and analyses. Heron 37, 1-79.

[15] Huang, M., Li, Y., 2013. X-ray tomography image-based reconstruction of microstructural finite element mesh models for heterogeneous materials. Computational Materials Science 67, 63-72. 
[16] Huang, Y. J., Yang, Z. J., Chen, X. W., Liu, G. H., 2016. Monte carlo simulations of meso-scale dynamic compressive behaviour of concrete based on x-ray computed tomography images. Journal of Impact Engineering 97, 102-115.

[17] Huang, Y. J., Yang, Z. J., Liu, G. H., Chen, X. W., 2016. An efficient fe-sbfe coupled method for mesoscale cohesive fracture modelling of concrete. Computational Mechanics $58,635-655$.

[18] Karihaloo, B. L., 1995. Fracture mechanics and structural concrete. Concrete Design and Construction Series. Longman Scientific \& Technical, Harlow, Essex, England.

[19] Kozicki, J., Tejchman, J., 2008. Modelling of fracture process in concrete using a novel lattice model. Grannular Matter 10, 377-388.

[20] Landis, E., Bolander, J., 2009. Explicit representation of physical prprocess in concrete fracture. Journal of Physics D: Applied Physics 42, 1-17.

[21] Legrain, G., Allais, R., Cartraud, P., 2011. On the use of the extended finite element method with quadtree/octree meshes. International Journal for Numerical Methods in Engineering 86, 717-743.

[22] Lian, W. D., Cartraund, G. L. L., 2013. Image-based computational homogenisation and localisation: Comparison between $\mathrm{x}$-fem level set and voxed-based approaches. Computational Mechanics 51, 279-293.

[23] López, C. M., Carol, I., Aguado, A., 2008. Meso-structural study of concrete fracture using interface eelement i: numerical model and tensile behaviour. Materials and Structures $41,583-599$.

[24] Monteiro, P., Maso, J., Olliver, J., 1985. The aggregate-mortar interface. Cement and Concrete Research 15, 953-958.

[25] Nitka, M., Tejchman, J., 2015. Modelling of concrete behaviour in uniaxial compression and tension with dem. Granular Matter 17, 145-164.

[26] Ooi, E. T., Man, H., Natarajan, S., Song, C., 2015. Adaptation of quadtree meshes in the scaled boundary finite element method for crack propagation modelling. Engineering Fracture Mechanics 144, 101-117.

[27] Pan, J., Feng, Y. T., Jin, F., Xu, Y., Sun, Q., Zhang, C., Owen, D. R. J., 2013. Mesoscale particle modeling of concrete deterioration caused by alkali-aggregate reaction. 
International Journal for Numerical and Analytical Methods in Geomechanics 37, 26902705.

[28] Petersson, P. E., 1981. Crack growth and development of fracture zone in plain concrete and similar materials. Technical Report TVBM-1006, Lund Institute of Technology.

[29] Reas, C., Fry, B., 2014. Processing: A Programming Handbook for Visual Designers and Artists (2nd Edition).

[30] Reddy, P., Montas, H. J., Samet, H., Shirmohammadi, A., 2001. A quadtree-based triangular mesh generation for finite element analysis of heterogeneous spatial media. ASAE Annual International Meeting 300 ((01-3072)), 1-25.

[31] Ren, W., Yang, Z. J., Sharma, R., Zhang, C., Withers, P. J., 2015. Two dimensional x-ray ct image bsed meso-scale fracture modelling of concrete. Engineering Fracture Mechanics 133, 24-39.

[32] Rodrigues, E. A., Manzoli, O. L., Jr, L. A. G. B., Bittencourt, T. N., 2016. 2dmesoscale concrete model for concrete based on the use of interface element with high aspect ratio. International Journal of Solids and Structures 94-95, 112-124.

[33] Roth, S. N., Leger, P., Soulaimani, A., 2015. A combined xfem-damage mechanics approach for concrete crack propagation. Computer Methods in Applied Mechanics and Engineering 283, 923-955.

[34] Saputra, A., Talebi, H., Tran, D., Birk, C., Song, C., 2017. Automatic image-based stress analysis by the scaled boundary finite element method. International Journal for Numerical Methods in Engineering 109, 697-738.

[35] Schlangen, E., Garboczi, E., 1997. Fracture simulations of concrete using lattice models: Computational aspects. Engineering Fracture Mechanics 57, 319-332.

[36] Schlangen, E., van Mier, J., 1992. Simple lattice model for numerical simulation of fracture of concrete materials and structures. Materials and Structures 25, 534-542.

[37] Shahbazi, S., Rasoolan, I., 2017. Meso-scale finite element modeling of non-homogeneous three-phase concrete. Case Studies in Construction Materials 6, 29-42.

[38] Shahbeyk, S., Hosseini, M., Yaghoobi, M., 2011. Mesoscale finite element prediction of concrete failure. Computational Material Science 50, 1973-1990. 
[39] Skarżyński, L., Tejchman, J., 2010. Calculations of fracture process zone on meso-scale in notched concrete beams subjected to three-point bending. European Journal of Mechanics A/Solids 29, 746-760.

[40] Snozzi, L., Gatuingt, F., Molinari, J. F., 2012. A meso-mechanical model for concrete under dynamic tensile and compressive loading. International Journal of Fracture 178, 179-194.

[41] Song, C., 2004. A matrix function solution for the scaled boundary finite element equation in statics. Computer Methods in Applied Mechanics and Engineering 193, 23252356.

[42] Song, C., Wolf, J. P., 1997. The scaled boundary finite element method - alias consistent infinitesimal finite element cell method for elastodynamics. Computer Methods in Applied Mechanics and Engineering 147, 329-355.

[43] Su, X. T., Yang, Z. J., Liu, G. H., 2010. Finite element modelling of complex 3d static and dynamic crack propagation using cohesive elements in abaqus. Acta Mechanica Solida Sinica 23, 271-282.

[44] Su, X. T., Yang, Z. J., Liu, G. H., 2010. Monte carlo simulation of complex cohesive fracture in random heterogeneous quasi-brittle materials: A 3d study. International Journal of Solids and Structures 47, 2336-2345.

[45] Suchorzewski, J., Tejchman, J., Nitka, M., 2017. Discrete element method ssimulation of fracture in concrete under uniaxial compression based on its real internal structure. International Journal of Damage Mechanics 0, 1-30.

[46] Tabarraei, A., Sukumar, N., 2005. Adaptive computations on conforming quadtree meshes. Finite Elements in Analysis and Design 41, 686-702.

[47] Trawiński, W., Bobiński, J., Tejchman, J., 2016. Two-dimensional simulations of concrete fracture at aggregate level with cohesive elements based on x-ray ct images. Engineering Fracture Mechanics 168, 204-226.

[48] Wang, X. F., Yang, Z. J., Yates, J. R., Jivkov, A. P., Zhang, C., 2015. Monte carlo simulations of mesoscale fracture modelling of concrete with random aggregates and pores. Construction and Building Materials 75, 35-45.

[49] Wang, Z. M., Huang, Y. J., Yang, Z. J., Liu, G. H., Wang, F., 2017. Efficient mesoscale homogenisation and statistical size effect analysis of concrete modelled by scaled boundary finite element polygons. Construction and Building Materials 151, 449-463. 
[50] Wang, Z. M., Kwan, A. K. H., Chan, H. C., 1999. Mesoscopic study of concrete i: generation of random aggregate structure and finite element mesh. Computers and Structures $70,533-544$.

[51] Wriggers, P., Moftah, S. O., 2006. Mesoscale models for concrete: Homogenisation and damage behaviour. Finite Elements in Analysis and Design 42, 623-636.

[52] Xu, X. P., Needleman, A., 1994. Numerical simulations of fast crack growth in brittle solids. Journal of the Mechanics and Physics of Solids 42, 1397-1434.

[53] Yaghoobi, A., Chorzepa, M. G., Kim, S. S., Durham, S. A., 2017. Mesoscale fracture analysis of multiphase cementitious composites using peridynamics. Materials 10, 162183.

[54] Yang, Z. J., Su, X. T., Chen, J. F., Liu, G. H., 2009. Monte carlo simulation of complex cohesive fracture in random heterogeneous quasi-brittle materials. International Journal of Solids and Structures 46, 3222-3234.

[55] Yang, Z. J., Xu, X. F., 2008. A heterogeneous cohesive model for quasi-brittle materials considering spatially varying random fracture properties. Computer Methods in Applied Mechanics and Engineering 197, 4027-4039.

[56] Young, P., Beresford-West, T., Coward, S., Notarberardino, B., Walker, B., Abdul-Aziz, A., 2008. An efficient approach to converting three-dimensional image datamodelshighly accurate computational models. Philosophical Transactions of the Royal Society of London A: Mathematical, Physical and Engineering Sciences 366, 3155-3173.

[57] Zhang, N., Guo, X., Zhu, B., Guo, L., 2012. A mesoscale model based on monte-carlo method for concrete fracture behaviour study. Science China Technological Sciences 55, 3278-3284. 